ARTICLE

DOI: $10.1038 / s 41467-017-00628-y$

OPEN

\title{
The autophagy initiator ULK1 sensitizes AMPK to allosteric drugs
}

Toby A. Dite ${ }^{1}$, Naomi X.Y. Ling ${ }^{1}$, John W. Scott ${ }^{2,3}$, Ashfaqul Hoque ${ }^{1}$, Sandra Galic ${ }^{2}$, Benjamin L. Parker ${ }^{4}$, Kevin R.W. Ngoei ${ }^{2}$, Christopher G. Langendorf², Matthew T. O'Brien², Mondira Kundu ${ }^{5}$, Benoit Viollet (1) ${ }^{6,7,8}$, Gregory R. Steinberg ${ }^{9}$, Kei Sakamoto ${ }^{10,11}$, Bruce E. Kemp (iD ${ }^{2,3}$ \& Jonathan S. Oakhill,3

AMP-activated protein kinase (AMPK) is a metabolic stress-sensing enzyme responsible for maintaining cellular energy homeostasis. Activation of AMPK by salicylate and the thienopyridone A-769662 is critically dependent on phosphorylation of Ser108 in the $\beta 1$ regulatory subunit. Here, we show a possible role for Ser108 phosphorylation in cell cycle regulation and promotion of pro-survival pathways in response to energy stress. We identify the autophagy initiator Unc-51-like kinase 1 (ULK1) as a $\beta 1$-Ser108 kinase in cells. Cellular $\beta 1$-Ser108 phosphorylation by ULK1 was dependent on AMPK $\beta$-subunit myristoylation, metabolic stress associated with elevated AMP/ATP ratio, and the intrinsic energy sensing capacity of AMPK; features consistent with an AMP-induced myristoyl switch mechanism. We further demonstrate cellular AMPK signaling independent of activation loop Thr172 phosphorylation, providing potential insight into physiological roles for Ser108 phosphorylation. These findings uncover new mechanisms by which AMPK could potentially maintain cellular energy homeostasis independently of Thr172 phosphorylation.

\footnotetext{
${ }^{1}$ Metabolic Signalling Laboratory, St Vincent's Institute of Medical Research, University of Melbourne, Melbourne, VIC, Australia. ${ }^{2}$ Protein Chemistry \& Metabolism, St Vincent's Institute of Medical Research, University of Melbourne, Melbourne, VIC, Australia. ${ }^{3}$ Mary MacKillop Institute for Health Research, Australian Catholic University, Melbourne, VIC, Australia. ${ }^{4}$ Charles Perkins Centre, School of Molecular Bioscience, The University of Sydney, Sydney, NSW, Australia. ${ }^{5}$ Department of Pathology, St Jude Children's Research Hospital, Memphis, TN, USA. ${ }^{6}$ INSERM, U1016, Institut Cochin, Paris, France. ${ }^{7}$ CNRS, UMR8104, Paris, France. ${ }^{8}$ Université Paris Descartes, Sorbonne Paris Cité, Paris, France. ${ }^{9}$ Divisions of Endocrinology and Metabolism, Department of Medicine, and Department of Biochemistry and Biomedical Sciences, McMaster University, Hamilton, ON, Canada. ${ }^{10}$ MRC Protein Phosphorylation and Ubiquitylation Unit, School of Life Sciences, University of Dundee, Scotland, UK. "11Present address: Nestlé Institute of Health Sciences SA, Lausanne, Switzerland. Toby A. Dite and Naomi X.Y. Ling contributed equally to this work. Correspondence and requests for materials should be addressed to J.S.O. (email: joakhill@svi.edu.au)
} 
T he evolutionarily conserved AMP-activated protein kinase (AMPK) is a key regulator of cellular and whole-body energy homeostasis that controls multiple branches of metabolism to redress energy imbalances caused by physiological and pathological processes ${ }^{1,2}$. AMPK senses increased cellular AMP/ATP ratio during periods of energy stress (hypoxia, nutrient deprivation, exercise) and protects the cell from these events by switching off energy-consuming anabolic pathways and switching on catabolic pathways to restore ATP levels. Multiple physiological processes are regulated by AMPK including autophagy, appetite control, mitochondrial biogenesis and cell growth, and proliferation. Consequently, extensive efforts have been made to develop AMPK-activating drugs for potential therapeutic use in treating metabolic diseases (type 2 diabetes, obesity, cardiovascular disease) and also cancer and inflammatory diseases.

The AMPK $\alpha \beta \gamma$ heterotrimer comprises an $\alpha$-catalytic subunit and regulatory $\beta$ - and $\gamma$-subunits. Multiple isoforms of each subunit exist in mammals $(\alpha 1 / 2, \beta 1 / 2, \gamma 1 / 2 / 3)$ and isoformspecific variations in tissue distribution, regulation, and function have been demonstrated. Both $\beta$-isoforms contain a carbohydrate-binding module (CBM) and are myristoylated at position Gly2, a modification that targets AMPK to intracellular membranes and is important for temporospatial regulation of AMPK signaling ${ }^{3,4} \cdot \gamma$-subunits possess three allosteric adenylate nucleotide-binding sites that bind ATP, ADP and AMP interchangeably, enabling AMPK to sense fluctuations in cellular energy state ${ }^{5-7}$.

In most instances, ligand-induced allosteric regulation of AMPK is governed by distinct phosphorylation events that either sensitize AMPK to nucleotides/drugs binding at $\gamma$-subunit sites (phosphorylation of Thr172 (pThr172) in the $\alpha$-subunit activation loop ${ }^{8-10}$ ), or small compounds binding at the ADaM (allosteric drug and metabolism) site (phosphorylation of $\beta$-Ser108 (pSer108) in the $\beta$-CBM ${ }^{11-13}$ ). An exception is synergistic activation of unphosphorylated AMPK when $\gamma$ - and ADaM sites are occupied simultaneously ${ }^{10,13}$. The ADaM site, a largely hydrophobic cavity formed between the $\alpha$-kinase domain small lobe and $\beta$-subunit CBM, was identified in crystal structures of
AMPK/drug complexes ${ }^{12,}{ }^{14}$. These structures revealed that the phosphate group of pSer108 forms electrostatic interactions with a2-kinase domain residues Thr21, Lys31, and Lys33, thereby stabilizing the $\mathrm{ADaM}$ site and explaining the role of pSer108 in mediating drug sensitization. The precise mechanism by which ADaM site drugs activate AMPK is not fully understood. pSer 108 is absolutely required for AMPK activation by A-769662 11,13 , salicylate ${ }^{15}$ and MT47-100 ${ }^{16}$, and increases by 40 -fold potency of the high affinity drug $991^{12}$. Using purified enzyme we further demonstrated that activation loop phosphorylation is dispensable for AMPK stimulation by A- $769662^{14}$. The well-defined character of the AMPK drug site, and its regulation through reversible phosphorylation, has led to speculation that synthetic activators (991, A-769662) and salicylate are mimicking an endogenous metabolite(s) that would be capable of sustaining AMPK signaling in the absence of pThr172, 17 .

Thr172 phosphorylation is considered a marker of AMPK activity; identification of upstream kinases, and the mechanisms underpinning pThr172 regulation, have been the subject of intense investigation over several decades. LKB1 and CaMKK2 $\left(\mathrm{Ca}^{2+} /\right.$ calmodulin-dependent protein kinase kinase 2) have been identified as in vivo Thr172 kinases ${ }^{18}$. Despite some conflicting evidence, current models of AMPK regulation by adenine nucleotides describe a tripartite mechanism in which ATP exchange for AMP and ADP at $\gamma$-sites (i) promotes Thr172 phosphorylation, (ii) suppresses pThr172 dephosphorylation, and (iii) (for AMP) allosterically activates Thr172-phosphorylated AMPK $^{3}, 5,6$. Hierarchical phosphorylation events in the $\alpha$-subunit Ser/Thr rich ST-loop (human $\alpha 1(472-525)$ ) have also been reported to negatively regulate pThr172, either by suppressing Thr172 phosphorylation ( $\alpha$-Ser487 auto-, Akt- or PKAphosphorylation ${ }^{1,}{ }^{2}$ ), or by promoting pThr172 dephosphorylation $\left(\alpha-T h r 479\right.$ phosphorylation by GSK3 $\left.{ }^{19}\right)$. Activity of the autophagy initiator Unc-51-like kinase (ULK), itself an AMPK substrate, is associated with reduced pThr172 via an uncharacterized negative feedback loop ${ }^{20}$.

In contrast, regulation and function of $\beta$-Ser 108 phosphorylation have been largely unexplored. Ser108 is highly conserved
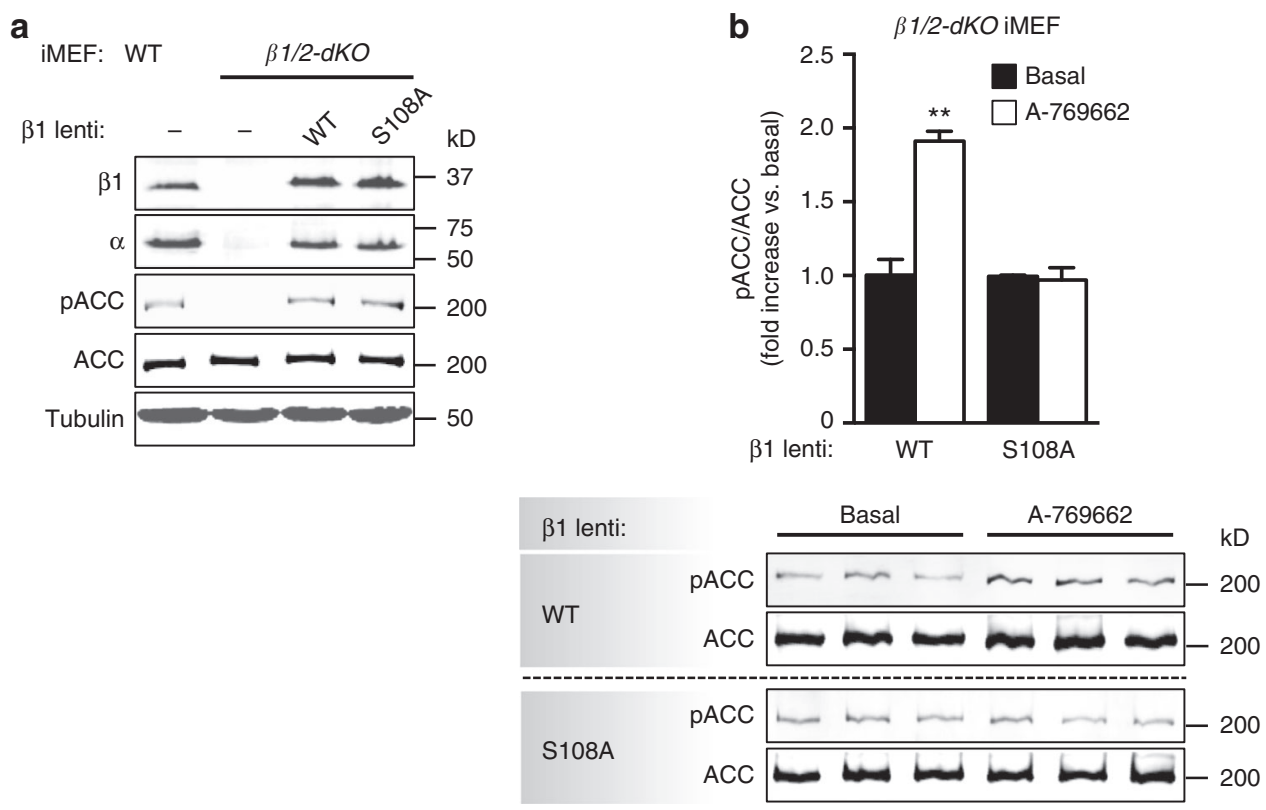

Fig. 1 A-769662 activation of cellular AMPK signaling is dependent on $\beta 1-p S e r 108$. a Reconstitution of basal AMPK signaling in AMPK $\beta 1 / 2$ double knockout ( $\beta 1 / 2-d K O)$ iMEFs by lentiviral transduction of AMPK $\beta 1$ WT or S108A mutant. b Immunoblots for pACC from $\beta 1 / 2$ - $d K O$ iMEFs-expressing $\beta 1$ WT or S108A mutant, stimulated with $20 \mu \mathrm{M}$ A-769662 for 90 min. $n=3$. Error bars, mean pACC fold change relative to basal \pm s.e.m. Statistical analysis was performed using unpaired two-tailed Student's $t$-test. ${ }^{\star \star} P<0.01$ indicates significant increase in $\mathrm{pACC}$ compared to basal 
a

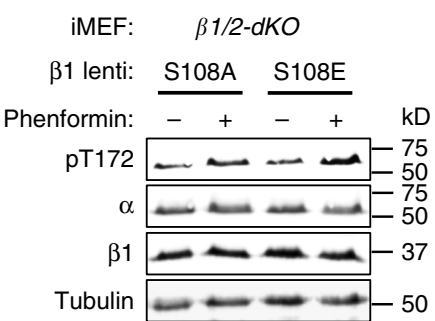

b $\quad \beta 1 / 2-d K O i M E F$

$(n=3)$
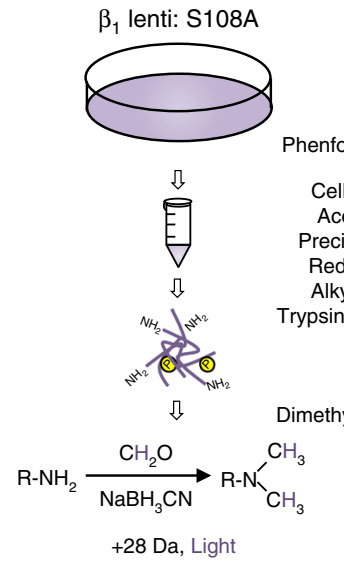

Phenformin, $1 \mathrm{~h}$

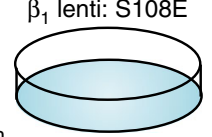

I

Mix (Medium:Light, 1:1)

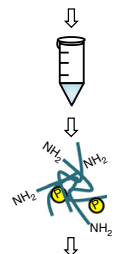

Acetone

Precipitation

imethyl labeling

$\sqrt{3}$

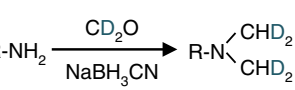

+32 Da, Medium

\ Run LC-MS/MS for global proteome

$\mathrm{TiO}_{2}$ phosphopeptide enrichment

ת

Run LC-MS/MS for phosphoproteome

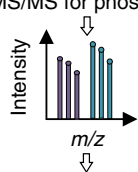

Raw data analysis, peptide identification,

protein assembly
C

OSB11_MOUSE (S179)

PAK2_MOUSE (S141, S152)

DOCK7_MOUSE (S452)

ANS1A MOUSE (S663)

PALM_MOUSE (T141, T145)

TB182 MOUSE(S1133)

TR150_MOUSE (S238, S243)

TCOF_MOUSE (S1191)

TCOF MOUSE (S593)

GLCE_MOUSE (S73)

IBTK_MOUSE (S1046)

ML12B_MOUSE (T19, S20)

CND1_MOUSE (S1320, S1323)

BIN1 MOUSE (S296)

NHRF1_MOUSE (S285)

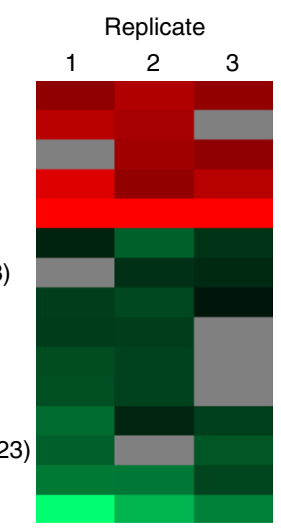

d IMEF: $\beta 1 / 2-d K O$

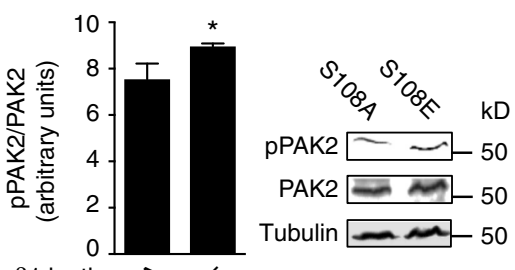

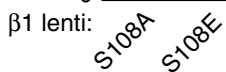

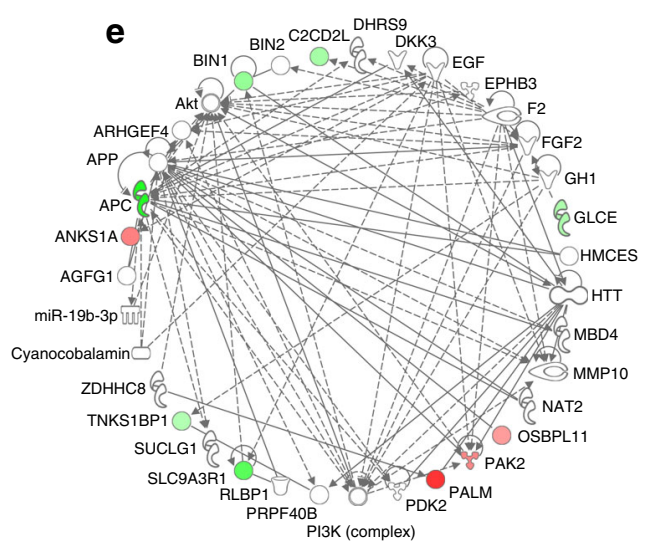

Fig. 2 Quantitative global and phosphoproteomic analysis uncovers cellular roles for $\beta 1$-pSer108. a Representative immunoblots for $\beta 1 / 2$ - $d K O$ iMEFs-expressing $\beta 1$ mutants S108A or S108E, stimulated with $2 \mathrm{mM}$ phenformin for $1 \mathrm{~h}$. $\mathbf{b}$ Workflow showing the stable isotope dimethyl labeling-based quantitative proteomic and phosphoproteomic approach. $\mathbf{c}$ Heatmap showing significantly perturbed cellular phosphoproteins and corresponding phosphosites. Red indicates increased, and green decreased, phosphorylation in $\beta 1-\mathrm{S} 108 \mathrm{E}$ compared to $\beta 1-\mathrm{S} 108 \mathrm{~A}$-expressing cells. Gray indicates missing phosphopeptide in that replicate. d Immunoblot/densitometry analysis confirming increased PAK2-Ser141 phosphorylation in $\beta 1$-S108E-expressing cells. $n=3$, representative immunoblot is shown. Error bars, mean PAK2-Ser141 phosphorylation (arbitrary units) \pm s.e.m. Statistical analysis was performed using unpaired two-tailed Student's $t$-test. ${ }^{*} P<0.05$ indicates significant increase in PAK2-pSer141 in S108E-expressing cells compared to S108Aexpressing cells. e "Cell cycle, connective tissue development and function, cellular movement" is one of the top networks associated with changes in phosphoproteome between S108A and S108E-expressing iMEFs, as identified by Ingenuity Pathway Analysis software

in eukaryotes and was identified as an autophosphorylation site in rat liver AMPK preparations ${ }^{21}$. Closer examination using kinase inactive (KI) AMPK expressed in COS-7 cells revealed that Ser108 is a cis-autophosphorylation site (dependent on intramolecular Thr172 phosphorylation) that is dephosphorylated following removal of the AMPK-activating stimulus. Thus, as for Thr172, Ser108 is largely unphosphorylated under basal conditions ${ }^{13}$. Identification of alternate upstream kinases for Ser108 would provide advances in two important areas: firstly, characterization of novel Ser108 kinases implicates their therapeutic modulation as a strategy to increase potency of AMPK-targeting drugs; secondly, feed-forward regulation by Ser108 kinases might denote the cellular processes under which concentrations of natural AMPK ligands become elevated. Here, we demonstrate that AMPK $\beta 1$-Ser108 is a substrate for ULK1 under conditions associated with elevated AMP. We also provide unambiguous demonstration of AMPK signaling independently of Thr172 phosphorylation. These findings underpin a ULK-mediated "ligand switch" model of AMPK allosteric control, in which the adenylate charge-sensing role of $\beta 1-A M P K$ is replaced by an ability to detect perturbations in endogenous metabolite(s) acting at the $\mathrm{ADaM}$ site.

\section{Results}

及1-pSer108 confers cellular AMPK drug sensitivity. The importance of Ser108 phosphorylation in sensitizing AMPK to $\mathrm{ADaM}$ site ligands has been well-characterized using purified 
AMPK enzyme in vitro, but whether this translates to cellular AMPK signaling has not been unequivocally demonstrated. To exclude signaling from endogenous AMPK we generated an immortalized mouse embryonic fibroblast (iMEF) cell line derived from mouse embryos harboring genetic deletion of both $\beta 1$ and $\beta 2$ isoforms ${ }^{22}(\beta 1 / 2-d K O)$. As expected, $\beta 1 / 2-d K O$ iMEFs were devoid of detectable AMPK $\alpha$ - or $\beta$-subunit expression and AMPK signaling as evidenced by lack of phosphorylation of the AMPK substrate ACC-Ser79 (Fig. 1a). Lentiviral-transduction of either FLAG-tagged wild-type (WT) or S108A mutant $\beta 1$ in $\beta 1 / 2$ $d K O$ iMEFs reconstituted expression of AMP-sensitive AMPK heterotrimers, and recovered phenformin-sensitive AMPK signaling in transduced cells (Fig. 1a and Supplementary Fig. 1a, b). Incubation with the direct AMPK agonist A-769662 $(20 \mu \mathrm{M})$ led to a significant increase (1.9-fold) in pACC-Ser79 in $\beta 1 / 2-d K O$ iMEFs-expressing WT $\beta 1$, but not the $\beta 1$ S108A mutant (Fig. 1b). A-769662-stimulation was mediated exclusively through the ADaM site, since phosphorylation of Thr172 was not increased at this dose (Supplementary Fig. 1c). These results confirm a requirement for Ser108 phosphorylation in drug activation of AMPK in cells.

Phosphoproteomic analysis hints at roles for $\boldsymbol{\beta} 1$-pSer108. To investigate the cellular fate of $\beta 1$-Ser108 phosphorylation, we performed a stable isotope dimethyl labeled-based quantitative proteomic and phosphoproteomic analysis using $\beta 1 / 2-d K O$ iMEFs, transduced with either $\beta 1$ mutant S108A or S108E (Fig. 2a). We previously showed that Glu at position 108 acts as an effective phosphomimetic, with regard sensitizing AMPK to activation by A-769662 $2^{13}$. Following $1 \mathrm{~h}$ phenformin $(2 \mathrm{mM})$ treatment, $\beta 1$ S108A and S108E transduced iMEF lysates were compared to trace out the changes in proteome and phosphoproteome (Fig. 2b). Fifteen cellular phosphoproteins showed significant changes in phosphorylation between S108A and S108E-expressing cells in our study (Fig. 2c, Supplementary Table 1) with no detectable differences in AMPK expression or phenformin-induced Thr172 phosphorylation (Fig. 2a, Supplementary Fig. 2a). For example, we identified increased phosphorylation of p21-activated kinase 2 (PAK2) on Ser141/Ser152, located in the kinase inhibitory domain, in phenformin-treated iMEFs-expressing $\beta 1$ S108E, whereas global proteome data showed no changes in PAK2 protein level (Supplementary Fig. 2b, c). Immunoblot analysis with a phosphospecific antibody confirmed significant increase in pPAK2-Ser141 in S108Eexpressing cells, validating our representative phosphoproteome data (Fig. 2d). Collectively, pathway and network analysis using ingenuity pathway analysis (IPA) identified "Cell cycle, connective tissue development and function, cellular movement" as

a

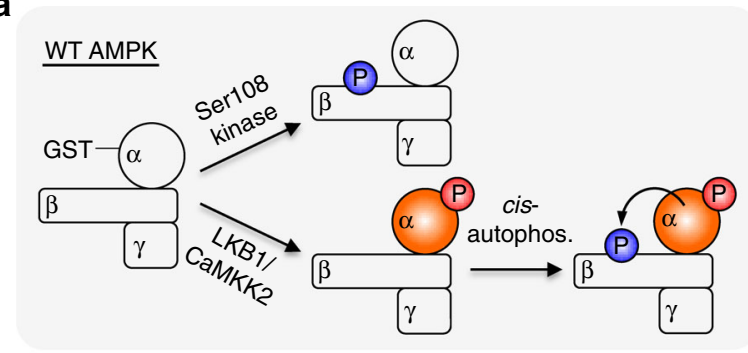

C
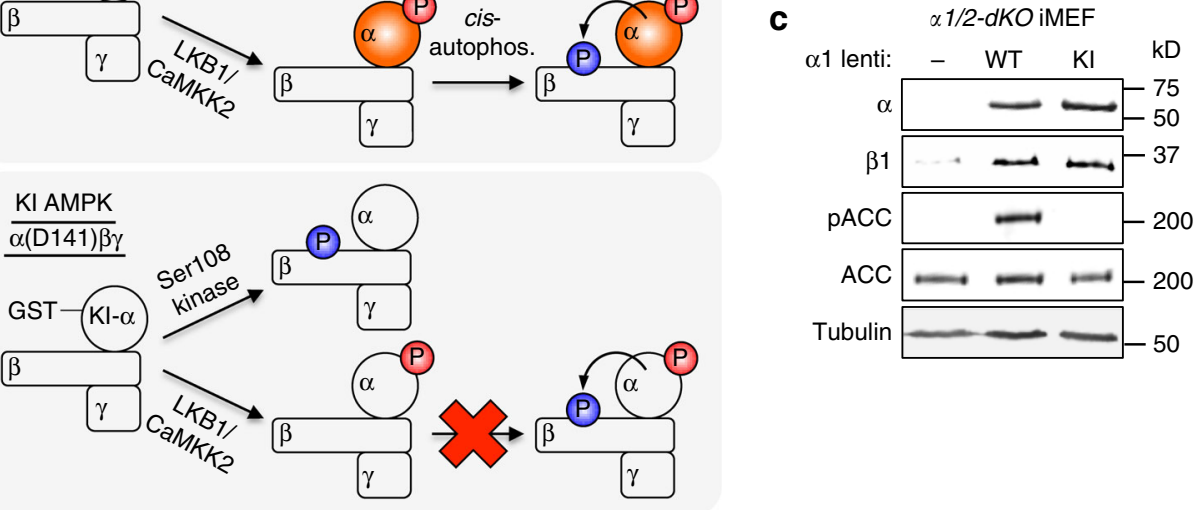

$$
\text { Tubulin }
$$
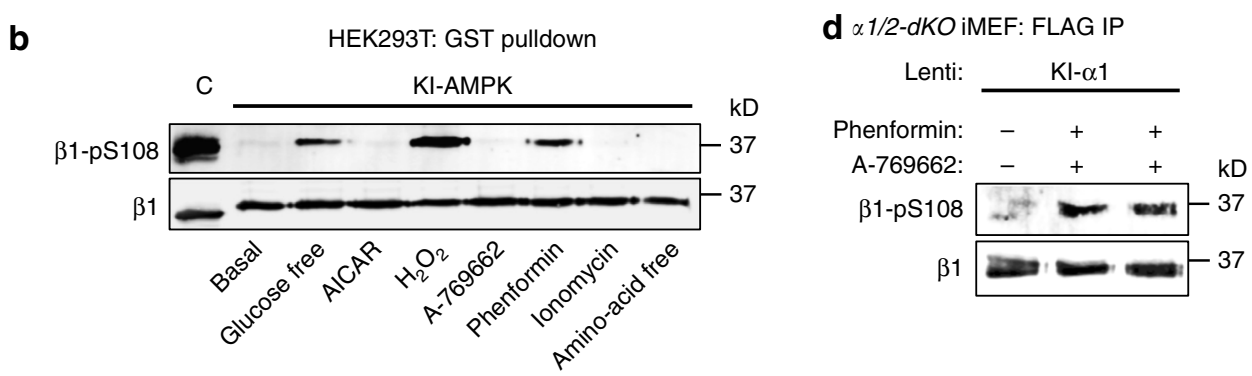

Fig. 3 1-Ser108 trans-phosphorylation occurs via an AMPK independent mechanism. a Rationale for employing kinase inactive (KI) AMPK to examine cellular Ser108 phosphorylation. $\beta 1-S e r 108$ phosphorylation (blue) can potentially be performed by an upstream kinase in both WT and KI $\alpha 1(D 141 A$ ) AMPK mutant. LKB1/CaMKK2-mediated phosphorylation of $\alpha$-Thr172 (red) activates WT AMPK (orange) leading to background Ser108 cis-autophosphorylation. This is excluded using KI AMPK, which can be phosphorylated on Thr172 but remains inactive. $\mathbf{b}$ Immunoblot for $\beta 1$-pSer108 in $\mathrm{KI}-\alpha 1 \beta 1 \gamma 1$ purified from HEK293T cells treated with AMPK-activating agents/conditions: glucose free (glucose-free DMEM $+10 \%$ serum, $4 \mathrm{~h}$ ), AICAR ( $2 \mathrm{mM}, 1 \mathrm{~h}), \mathrm{H}_{2} \mathrm{O}_{2}(1 \mathrm{mM}, 45 \mathrm{~min}), \mathrm{A}-769662(300 \mu \mathrm{M}, 1 \mathrm{~h})$, phenformin ( $\left.2 \mathrm{mM}, 1 \mathrm{~h}\right)$, ionomycin $(2.5 \mu \mathrm{M}, 15 \mathrm{~min})$ and amino-acid free (EBSS medium, $\left.4 \mathrm{~h}\right)$. $n=3$, representative immunoblots shown. C: Bacterial expressed, CaMKK2-treated $\alpha 1 \beta 1 \gamma 1$ standard. c Reconstitution of basal AMPK signaling in AMPK $\alpha 1 / 2$ double knockout ( $\alpha 1 / 2-d K O)$ iMEFs by lentiviral transduction of AMPK $\alpha 1$ WT, but not the KI mutant. d Immunoblot for $\beta 1-p S e r 108$ from $\alpha 1 / 2-d K O$ iMEFs-expressing KI- $\alpha 1$, stimulated with $2 \mathrm{mM}$ phenformin for $1 \mathrm{~h} . n=3$, representative immunoblots shown 

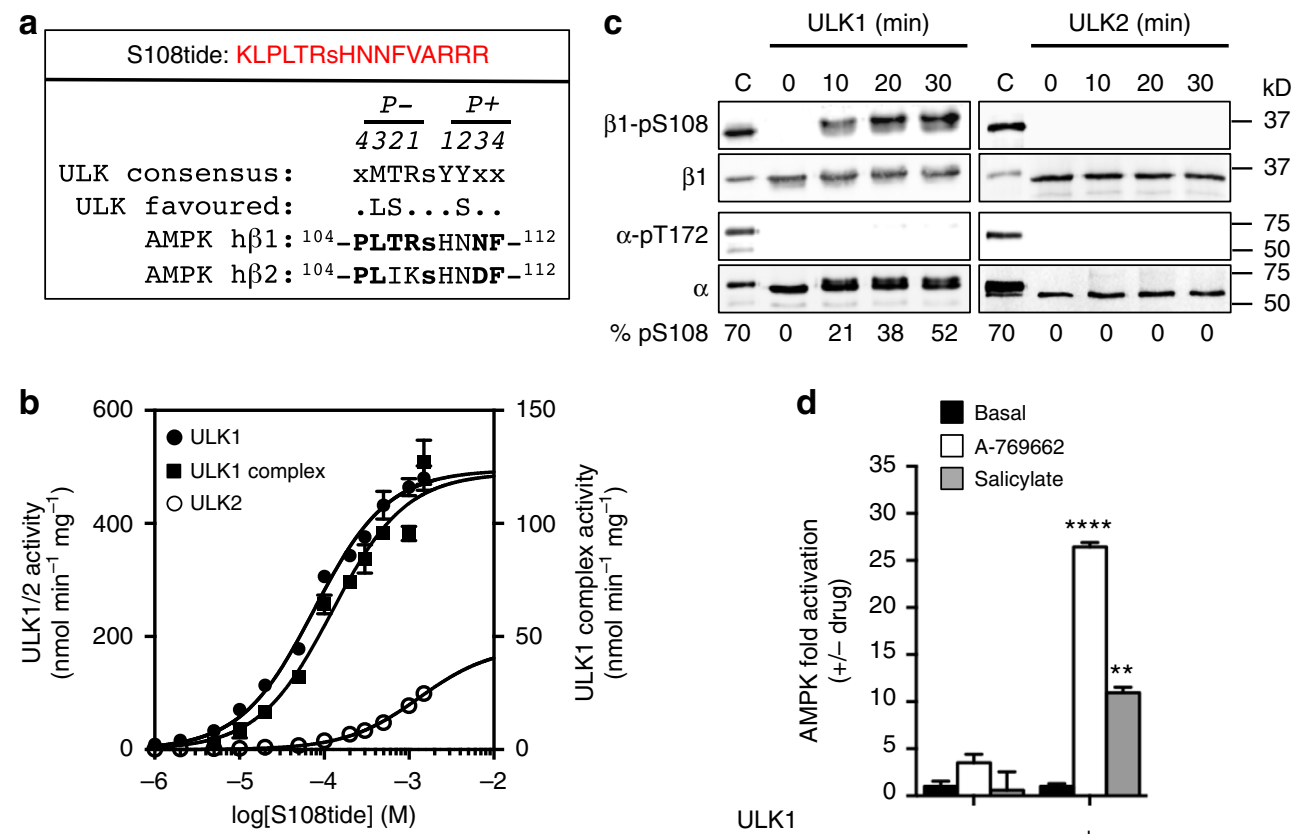

\begin{tabular}{|lc|}
\hline & $K_{\mathrm{m}}$ (mean \pm s.e.m. $)$ \\
\hline ULK1 & $75.8 \pm 5.6 \mu \mathrm{M}$ \\
ULK1 complex & $123.6 \pm 16.3 \mu \mathrm{M}$ \\
ULK2 & $1309.0 \pm 166.8 \mu \mathrm{M}$ \\
\hline
\end{tabular}

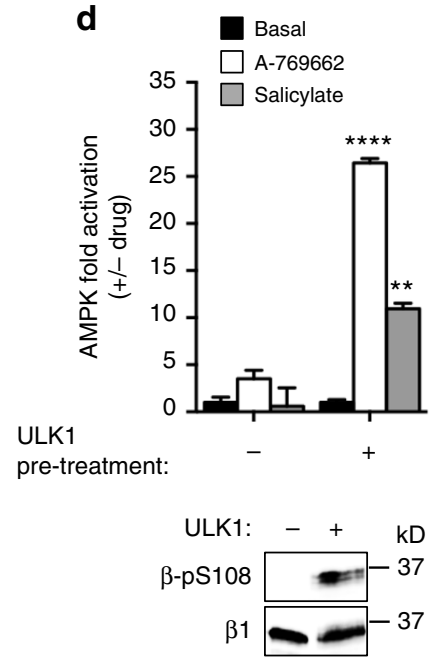

Fig. 4 ULK1 phosphorylates $\beta 1$-Ser108 in vitro. a Sequence alignment of the ULK consensus motif/favorable substitutions ${ }^{23}$ with human AMPK $\beta 1$ - and $\beta 2$-residues 104-112 (Ser108 in lower case). $x$ denotes positions with no demonstrated preference. Preferred/favored consensus $\beta$-residues are in bold. Sequence of the synthetic peptide S108tide is shown in red. b Dose curve of S108tide phosphorylation by ULK1 and ULK2 (plotted to left $y$-axis), and ULK1/ FIP200/Atg13 complex (plotted to right $y$-axis). $n=3$. Error bars, mean activity \pm s.e.m. c Immunoblots for $\beta 1$-pSer108 and $\alpha$-pThr172 in bacterial-expressed $\mathrm{KI}-\alpha 1 \beta 1 \gamma 1$ phosphorylated with ULK1 (left) or ULK2 (right) for 30 min. $n=3$, representative immunoblots shown. C: CaMKK2-treated $\alpha 1 \beta 1 \gamma 1$ control. d Activity of ULK1-phosphorylated $\alpha 1(\mathrm{C} 176 \mathrm{~S}) \beta 1 \gamma 1$ in the presence of $20 \mu \mathrm{M} \mathrm{A-769662}$ or $10 \mathrm{mM}$ salicylate. $n=3$, representative immunoblots of pSer108 in assayed AMPK preparations are shown. Error bars, mean fold AMPK activation relative to ULK1-untreated \pm s.e.m. Statistical analyses were performed using one way ANOVA with post hoc Dunnett's multiple comparison test. ${ }^{\star \star} P<0.01,{ }^{\star \star \star \star} P<0.0001$ indicate significant increase in AMPK activation compared to ULK1-untreated

one of the top networks associated with these perturbed cellular phosphoproteins (Fig. 2e).

及1-Ser108 can be phosphorylated in trans. We previously demonstrated that AMPK $\beta 1$-Ser108 is cis-autophosphorylated via an intramolecular mechanism reliant on prior phosphorylation of $\alpha$-Thr172 13 . This implies that, in the absence of alternate signaling, aberrant phosphatase regulation or conditions leading to trans-autophosphorylation, stoichiometries of pSer108 and pThr172 are intrinsically linked and, for the most part, equivalent. To interrogate this model we screened AMPK-activating conditions/agents under which Ser108 becomes a substrate for a trans-phosphorylation event in cells. To prevent background cissignaling we expressed a GST-fusion of the KI AMPK mutant $\alpha 1$ (D141A) $\beta 1 \gamma 1$ in HEK293T cells. This complex is a suitable substrate for Thr172-phosphorylating kinases LKB1 and CaMKK2, but does not undergo Ser108 cis-autophosphorylation (Fig. 3a). Glucose starvation, or incubation with $\mathrm{H}_{2} \mathrm{O}_{2}$ or phenformin, each induced phosphorylation at Ser108 (Fig. 3b). Incubation with ionomycin (activator of CaMKK2 signaling), AICAR (5-aminoimidazole-4-carboxamide ribonucleotide) or A-769662, or amino-acid deprivation, each failed to elicit Ser108 phosphorylation at the single experimental time point used. We used $\alpha 1 / 2-d K O$ iMEFs $^{23}$ to exclude the possibility that Ser108 is transautophosphorylated in phenformin-treated cells. Lentiviraltransduction of $\alpha 1 / 2-d K O$ iMEFs with FLAG-tagged WT- or
KI- $\alpha 1$ reconstituted AMPK expression; as expected only WT $\alpha 1$ transduction recovered AMPK signaling (Fig. 3c). However, phenformin induced phosphorylation of Ser108 in $\alpha 1 / 2-d K O$ iMEFs transduced with KI- $\alpha 1$, which was not increased by additional incubation with $100 \mu \mathrm{M}$ A-769662 (Fig. 3d). Our results demonstrate that, under certain metabolic stress conditions, Ser108 is a substrate for a kinase(s) other than AMPK autophosphorylation.

ULK1 phosphorylation of $\beta 1-S e r 108$ induces drug sensitivity. To identify upstream kinases for $\beta 1$-Ser108 we screened a synthetic peptide corresponding to AMPK $\beta 1(102-114)$ (S108tide) (Fig. 4a) against a panel of 284 Ser/Thr kinases. $92 \%$ of the kinases screened yielded low activities against S108tide ( $<10 \%$ vs. top hit). Several kinases from diverse groups demonstrated comparable $(>35 \%)$ specific activity relative to AMPK $\alpha 1 \beta 1 \gamma 1$, e.g., BRSK1/2, NEK2/9, TAOK1 (Supplementary Table 2). Among positive hits was ULK1, which is in accordance with identity of the sequence surrounding $\beta 1$-Ser108 to the consensus motif for substrates of this kinase ${ }^{24}$, particularly Leu, Thr, and Arg directly N-terminal to Ser108 (positions P-3, P-2, and P-1, respectively) (Fig. 4a). We performed a more detailed analysis and phosphorylated the S108tide substrate using purified FLAGULK1, either alone $\left(k_{\mathrm{cat}} / K_{\mathrm{m}} 1.23 \times 10^{-2} / \mathrm{s} / \mu \mathrm{M}\right)$ or complexed to interaction partners FIP200 and $\operatorname{Atg} 13\left(k_{\text {cat }} / K_{\mathrm{m}} 1.86 \times 10^{-3} / \mathrm{s} /\right.$ $\mu \mathrm{M}$ ) (Fig. 4b and Supplementary Fig. 3a). ULK2 demonstrated 

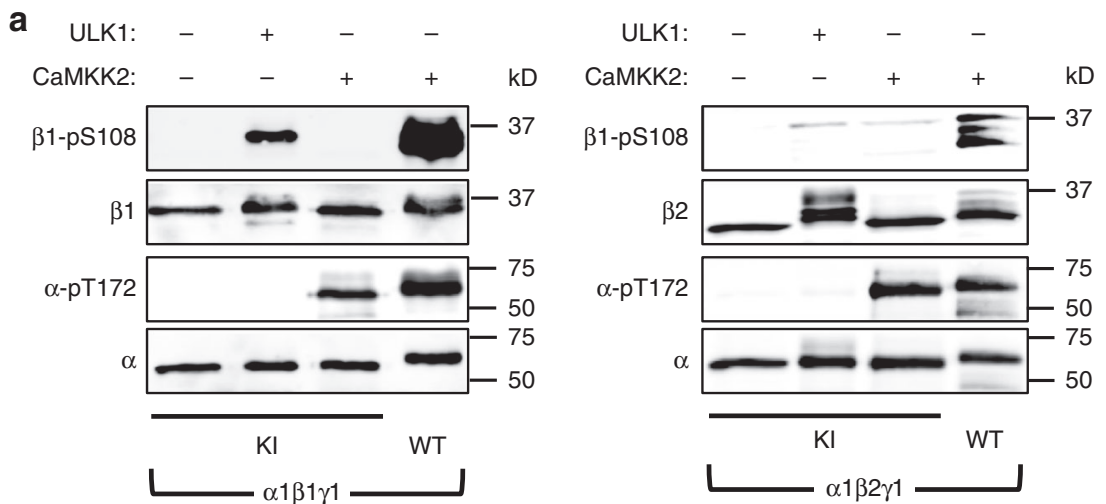

b
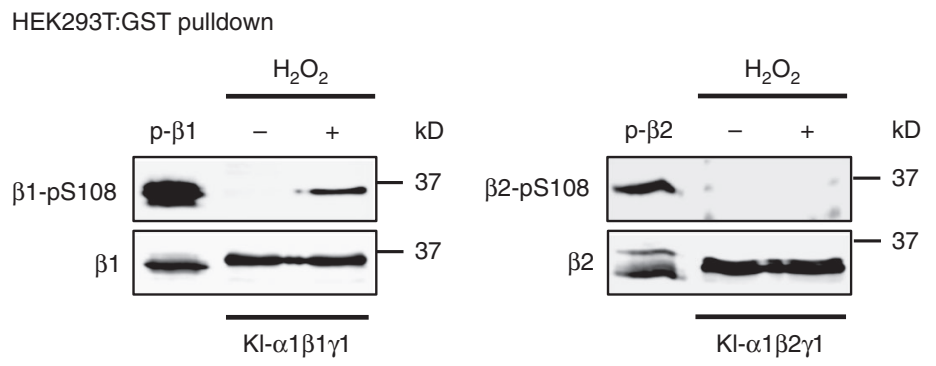

Fig. 5 ULK1 phosphorylation of Ser108 is specific for the AMPK $\beta 1$ isoform. a Immunoblots for $\beta 1$-pSer108, $\beta 2$-pSer108, and $\alpha$-pThr172 in bacterialexpressed KI- $\alpha 1 \beta 1 \gamma 1$ (left) or KI- $\alpha 1 \beta 2 \gamma 1$ (right) phosphorylated with ULK1 or CaMKK2 for 30 min. Controls: CaMKK2-treated WT $\alpha 1 \beta 1 \gamma 1$ (left) or $\alpha 1 \beta 2 \gamma 1$ (right). b Immunoblots for $\beta$-pSer108 in $\mathrm{KI}-\alpha 1 \beta 1 \gamma 1$ (left) or KI- $\alpha 1 \beta 2 \gamma 1$ (right) purified from HEK293T cells stimulated with $1 \mathrm{mM} \mathrm{H}_{2} \mathrm{O}_{2}$ for 45 min. Controls: $p-\beta 1$ and $p-\beta 2$, CaMKK2-treated WT $\alpha 1 \beta 1 \gamma 1$ and $\alpha 1 \beta 2 \gamma 1$, respectively. In both panels $n=3$, representative immunoblots shown

reduced efficiency in phosphorylating S108tide $\left(k_{\text {cat }} / K_{\mathrm{m}}\right.$ $2.63 \times 10^{-4} / \mathrm{s} / \mu \mathrm{M}$ ), compared to ULK1 (Fig. 4b). We examined ULK phosphorylation of the AMPK heterotrimer using purified KI- $\alpha 1 \beta 1 \gamma 1$ expressed in bacteria, since we previously found the WT complex extracted from this source is autophosphorylated at $\beta 1$-Ser108 with $\sim 60 \%$ stoichiometry despite lacking pThr $172^{13}$. ULK1 phosphorylated $\beta 1$-Ser108, but not $\alpha 1-$ Thr172, in KI-AMPK, whereas we did not detect phosphorylation of either residue by ULK2 (Fig. 4c). MS/MS analysis confirmed ULK1-phosphorylation of Ser108 in KI- $\alpha 1 \beta 1 \gamma 1$ (Supplementary Fig. 3b, c).

We found that expression of the mutant $\alpha 1(\mathrm{C} 176 \mathrm{~S}) \beta 1 \gamma 1$ in bacteria produced AMPK that was devoid of pSer108 (Supplementary Fig. 3d) yet retained full allosteric regulatory mechanisms, such as A-769662/AMP and A-769662/C2 synergistic activation (Supplementary Fig. 3e) ${ }^{10}, 13$, or individual A-769662 (Supplementary Fig. 3f) and AMP (Supplementary Fig. 3g) activation following phosphorylation by CaMKK2. One explanation is that pThr172-independent basal activity, and hence Ser108 autophosphorylation, is induced by modification of Cys176 in the activation loop, occurring as a result of oxidative stress during AMPK expression in bacteria. Redox sensitive mechanisms are known to regulate receptor tyrosine kinase ${ }^{25}$ and a variety of Ser/Thr kinases including CaMK2, PKA and $\mathrm{PKC}^{26-28}$. The $\alpha 1$ (C176S) $\beta 1 \gamma 1$ construct allowed us to investigate the effect of ULK phosphorylation on ligand-mediated regulation of AMPK without the need for prior and extensive phosphatase treatment. We found that ULK pre-treatment sensitized purified AMPK $\alpha 1$ (C176S) $\beta 1 \gamma 1$ to activation by A-769662 or salicylate (Fig. 4d). We detected ULK1-phosphorylation at several other AMPK $\alpha 1, \beta 1$, and $\gamma 1$ sites (Supplementary Fig. 3c). These included $\gamma 1$ residues Ser261 and Ser270, located in proximity to nucleotide site 3, which is important for AMP allosteric regulation ${ }^{3,7}$. However, AMP allosteric activation of WT $\alpha 1 \beta 1 \gamma 1$ was not significantly affected by pre-treatment with ULK1 (Supplementary Fig. 3g).
ULK1 phosphorylation of $\beta$-Ser108 is specific to AMPK $\beta 1$. Ser108 is conserved between mammalian AMPK $\beta$-isoforms 1 and 2, however identity of the $\beta 2$ sequence to the ULK consensus/ favored motif is restricted to Leu at P-3 (Fig. 4a). ULK1 was previously shown to phosphorylate multiple AMPK $\beta 2$ residues in vitro, but not Ser $108^{20}$. To confirm $\beta$-isoform specificity of ULK1 we generated a phosphospecific antibody to $\beta 2$-pSer108 (Supplementary Fig. 4). Incubation of WT $\alpha 1 \beta 1 \gamma 1$ or $\alpha 1 \beta 2 \gamma 1$ with CaMKK2 resulted in Thr172 phosphorylation and subsequent Ser108 autophosphorylation (Fig. 5a). ULK1 treatment of KI- $\alpha 1 \beta 2 \gamma 1$ resulted in reduced electrophoretic migration of $\beta 2$, indicative of multiple phosphorylation events. However, we did not detect $\beta 2$-pSer108 by immunoblot following ULK1 treatment (Fig. 5a, right panel). Furthermore, exposure of HEK293T cells to $\mathrm{H}_{2} \mathrm{O}_{2}$ failed to induce Ser108 phosphorylation in KI- $\alpha 1 \beta 2 \gamma 1$ (Fig. 5b, right panel).

及1-Ser108 is a cellular substrate for ULK. We examined whether ULK phosphorylates $\beta 1$-Ser108 in HEK293T cells. Initially, we used the highly selective, ULK1 small molecule inhibitor SBI-0206965 (termed 6965) ${ }^{24}$. We found that pre-incubation of transfected HEK293T cells with $10 \mu \mathrm{M}$ 6965, a concentration previously shown to have no effect on AMPK signaling ${ }^{24}$, significantly reduced $\mathrm{H}_{2} \mathrm{O}_{2^{-}}$(Fig. 6a) and phenformin- (Fig. 6b) induced phosphorylation of Ser108 in KI-AMPK $\alpha 1 \beta 1 \gamma 1$, relative to 6965 untreated cells. Treatment with 6965 caused a significant increase in pThr172 in KI- $\alpha 1 \beta 1 \gamma 1$ in response to both $\mathrm{H}_{2} \mathrm{O}_{2}$ and phenformin (Fig. 6a, b); this is consistent with a role for ULK1 as a negative regulator of Thr172 phosphorylation.

We investigated regulation of Ser108 phosphorylation in iMEFs in which both ULK1 and ULK2 had been genetically deleted $(u l k 1 / 2-d K O)^{29}$ (Supplementary Fig. 5). Under basal conditions, pSer108 in endogenous AMPK was significantly higher in both WT and ulk1/2-dKO iMEFs compared to 
HEK293T cells, despite no detectable increase in pThr172 (Fig. 6c). We bypassed this high basal signal in iMEFs by examining pSer108 in KI-AMPK, expressed by lentiviral transduction of a FLAG-tagged KI- $\alpha 1(\mathrm{D} 141 \mathrm{~A})$ mutant and isolated with endogenous $\beta$ - and $\gamma$-subunits by FLAG immunoprecipitation. KI-AMPK expressed in iMEFs was devoid of basal pSer108 (Fig. 6d). Phenformin-induced phosphorylation of Ser108 was significantly decreased $(>90 \%)$ in KI-AMPK from $u l k 1 / 2-d K O$ iMEFs, compared to WT iMEFs (Fig. 6d). Combined, these results confirm $\beta 1$-Ser108 as a cellular substrate for ULK1.

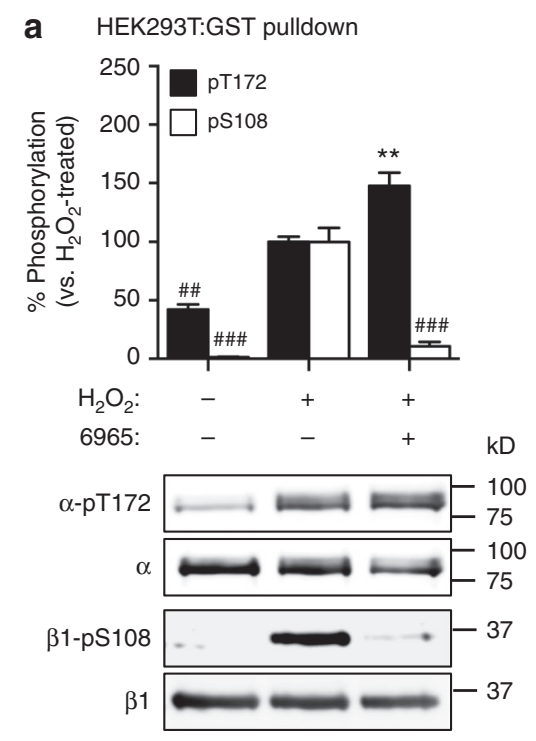

b HEK293T:GST pulldown
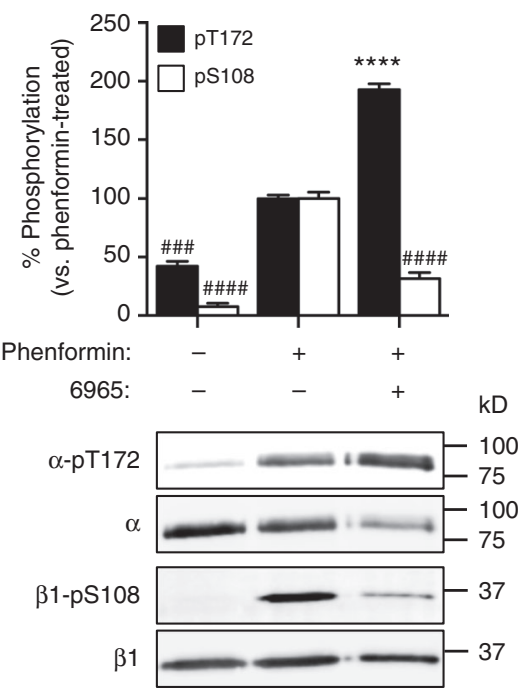

C Endogenous AMPK
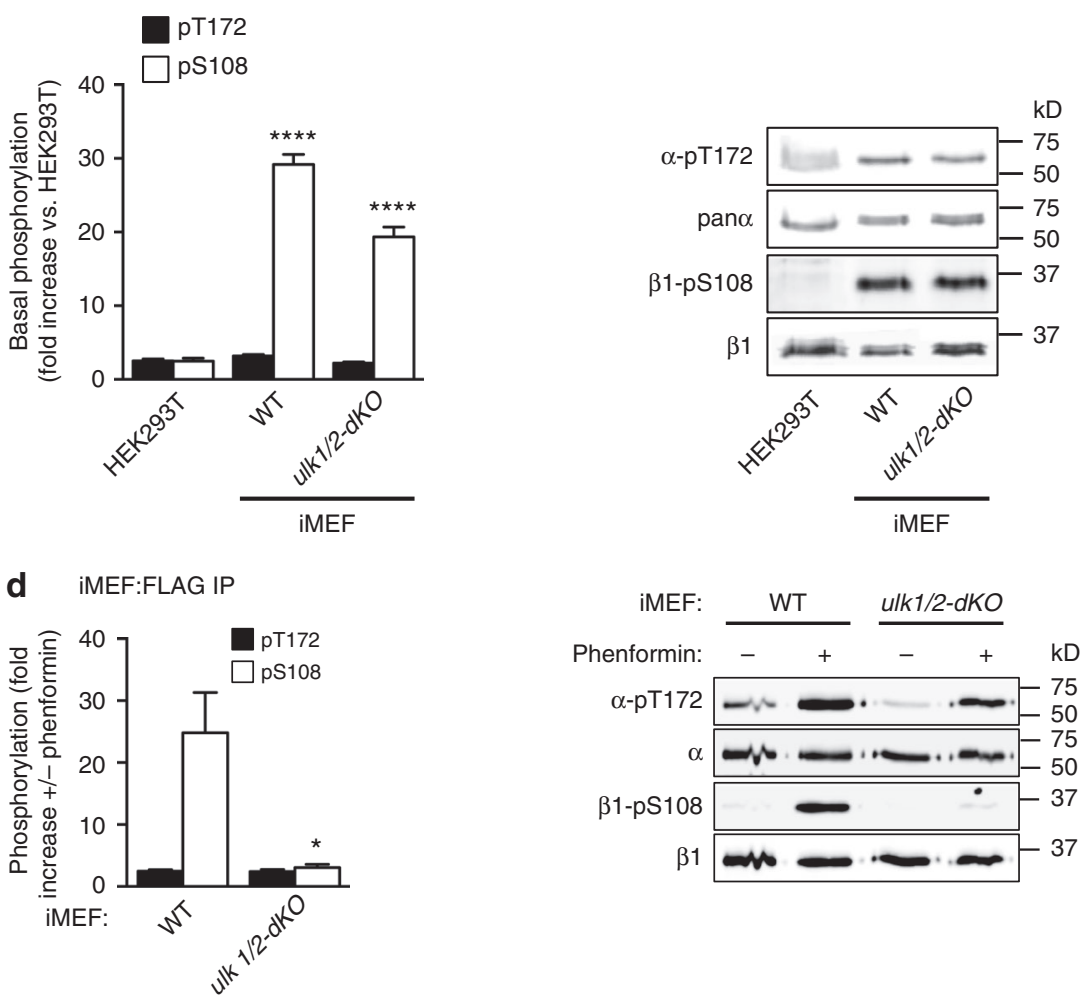

Fig. 6 ULK phosphorylates $\beta 1$-Ser108 in cells. Statistical analyses were performed using one-way ANOVA with post hoc Dunnett's multiple comparison

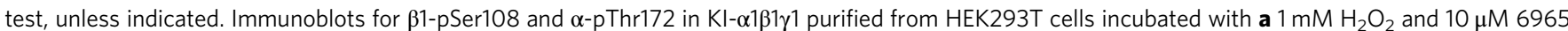
for $45 \mathrm{~min}$, or $\mathbf{b} 2 \mathrm{mM}$ phenformin and $10 \mu \mathrm{M} 6965$ for $1 \mathrm{~h} . \mathrm{n}=3$, representative immunoblots shown. Error bars, mean \% phosphorylation relative to $\mathrm{H}_{2} \mathrm{O}_{2}{ }^{-}$

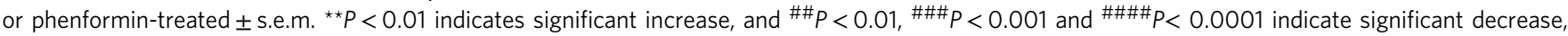
compared to $\mathrm{H}_{2} \mathrm{O}_{2}$ - or phenformin-treated. c Immunoblots for $\beta 1$-pSer108 and $\alpha$-pThr172 in lysates from HEK293T cells, WT or ulk1/2-dKO iMEFs incubated in $25 \mathrm{mM}$ glucose DMEM + 10\% serum. $n=3$ individual cultures per cell line, representative immunoblots shown. Error bars, mean fold increase in phosphorylation relative to HEK293T cells \pm s.e.m. ${ }^{\star \star \star \star} P<0.0001$ indicates significant increase in phosphorylation compared to HEK293T cells. d Immunoblots for $\beta 1$-pSer108 and $\alpha$-pThr172 in KI- $\alpha 1$ AMPK purified from WT or ulk1/2-dKO iMEFs stimulated with $2 \mathrm{mM}$ phenformin for $1 \mathrm{~h}$. $n=3$, representative immunoblots shown. Error bars, mean fold increase in phosphorylation relative to basal \pm s.e.m. Statistical analysis was performed using unpaired two-tailed Student's $t$-test. ${ }^{\star} P<0.05$ indicates significant decrease compared to WT iMEFs 


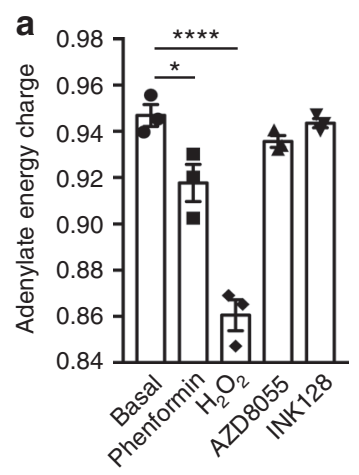

C

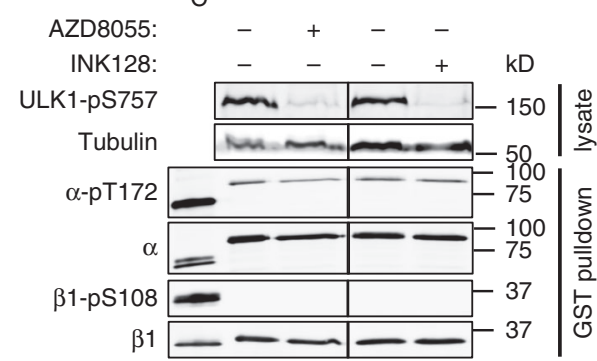

b

HEK293T:GST pulldown

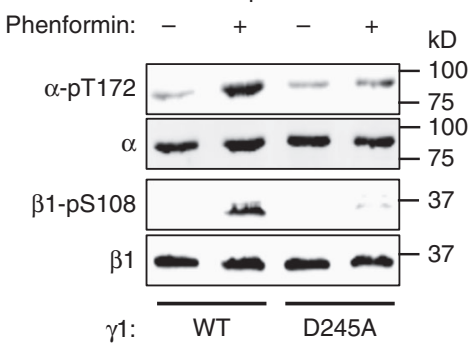

d HEK293T:GST pulldown

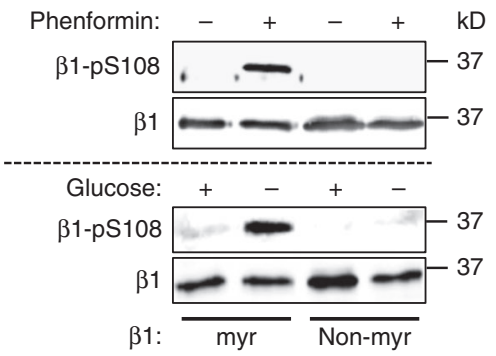

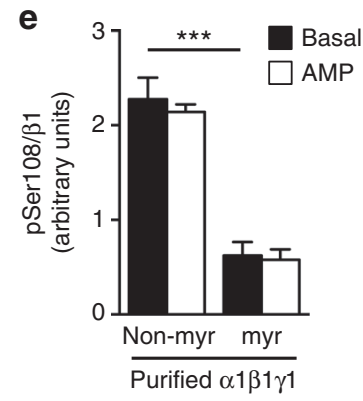

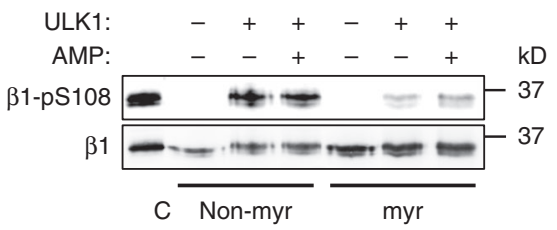

Fig. 7 An AMP-myristoyl switch triggers ULK1 phosphorylation of $\beta 1$-Ser108. a Adenine nucleotides extracted from HEK293T cells incubated with phenformin $(2 \mathrm{mM}, 1 \mathrm{~h}), \mathrm{H}_{2} \mathrm{O}_{2}(1 \mathrm{mM}, 45 \mathrm{~min}), \mathrm{AZD} 8055(1 \mu \mathrm{M}, 1 \mathrm{~h})$, or INK128 (1 $\left.1 \mathrm{M}, 1 \mathrm{~h}\right)$ were quantitated by mass spectrometry. Adenylate energy charge was calculated as described in Online Methods. $n=3$. Error bars, mean adenylate energy charge \pm s.e.m. Statistical analyses were performed using one-way ANOVA with post hoc Dunnett's multiple comparison test. ${ }^{\star \star \star \star} P>0.001,{ }^{\star} P<0.05$ indicate significant decrease in mean adenylate energy charge compared to basal. b Immunoblots for $\beta 1$-pSer108 and $\alpha$-pThr172 in KI- $\alpha 1 \beta 1 \gamma 1$ or KI- $\alpha 1 \beta 1 \gamma 1(\mathrm{D} 245 \mathrm{~A})$ purified from HEK293T cells stimulated with $2 \mathrm{mM}$ phenformin for 1 h. $n=3$, representative immunoblots shown. c Immunoblots for ULK1-pSer757, and $\beta 1$-pSer108 and $\alpha$-pThr172 in KI- $\alpha 1 \beta 1 \gamma 1$ purified from HEK293T cells incubated with $1 \mu \mathrm{M}$ mTOR inhibitors AZD8055 or INK128 for $1 \mathrm{~h} . n=3$, representative immunoblots shown. C: Bacterial expressed, CaMKK2-treated $\alpha 1 \beta 1 \gamma 1$ standard. $\mathbf{d}$ Immunoblots for $\beta 1$-pSer108 in KI- $\alpha 1 \beta 1 \gamma 1$ (myr) or KI- $\alpha 1 \beta 1(\mathrm{G} 2 \mathrm{~A}$ ) $\gamma 1$ (non-myr) purified from HEK293T cells incubated with $2 \mathrm{mM}$ phenformin for $1 \mathrm{~h}$ (upper) or glucose free medium for $4 \mathrm{~h}$ (lower). $n=3$, representative immunoblots shown. e Immunoblot for $\beta 1$-pSer108 in bacterial-expressed, non-myristoylated (non-myr) or myristoylated (myr) KI- $\alpha 1 \beta 1 \gamma 1$ phosphorylated with ULK1 for 30 min in the presence of $100 \mu M$ AMP. $n=3$, representative immunoblots shown. C: CaMKK2-treated $\alpha 1 \beta 1 \gamma 1$ standard. Error bars, mean increase in $\beta 1$-pSer108 relative to ULK1-untreated \pm s.e.m. Statistical analysis was performed using unpaired two-tailed Student's $t$-test. ${ }^{\star \star \star} P<0.001$ indicates significant decrease in $\beta 1$-pSer108 relative to non-myristoylated AMPK

An AMP myristoyl switch triggers $\beta 1$-Ser108 transphosphorylation. $\mathrm{H}_{2} \mathrm{O}_{2}$ and phenformin indirectly activate AMPK through perturbation of adenine nucleotide ratios (increased AMP/ATP ratio, reduced adenylate charge). We found that $\mathrm{H}_{2} \mathrm{O}_{2}$ and phenformin treatments of HEK293T cells that induced Ser108 phosphorylation (Fig. 3b) also produced significant falls in adenylate energy charge (AEC), with $\mathrm{H}_{2} \mathrm{O}_{2}$ incubation having the greater effect (AMP/ATP ratios: basal $0.0093 \pm$ 0.0015 ; phenformin $0.0177 \pm 0.0041 ; \mathrm{H}_{2} \mathrm{O}_{2} \quad 0.0418 \pm 0.0033$ ) (Fig. 7a). Therefore, we investigated whether elevated AMP was a requirement for phosphorylation of Ser 108 by ULK1. Phenformin treatment of HEK293T cells failed to induce Ser108 phosphorylation in KI- $\alpha 1 \beta 1 \gamma 1$ carrying a mutation in the $\gamma 1$ nucleotide site $3(\gamma 1-\mathrm{D} 245 \mathrm{~A})$ that renders AMPK insensitive to stimulation by
$\mathrm{AMP}^{3}$ (Fig. 7b). ULK1 can also be activated in response to small molecule mTOR inhibitors (AZD8055, INK128) that suppress mTOR-mediated phosphorylation at the ULK1 inhibitory site Ser757 24 . Incubation of HEK293T cells with $1 \mu \mathrm{M}$ AZD8055 or INK128 induced almost complete loss of ULK-pSer757, without significantly affecting adenylate charge or stimulating phosphorylation of Ser108 in KI- $\alpha 1 \beta 1 \gamma 1$ expressed in these cells (Fig. 7a, c). These data indicate that ULK1 phosphorylation of $\beta 1$-Ser108 in cells requires a reduction in adenylate charge and is dependent on the AMP-sensing abilities of AMPK.

$\mathrm{N}$-terminal myristoylation of $\beta$-Gly 2 plays important roles in AMPK temporospatial regulation, being required for AMPstimulation of Thr172 phosphorylation by upstream kinases and metabolic stress-induced co-localization of AMPK to both 
a $\alpha 1 / 2-d K O$ iMEF
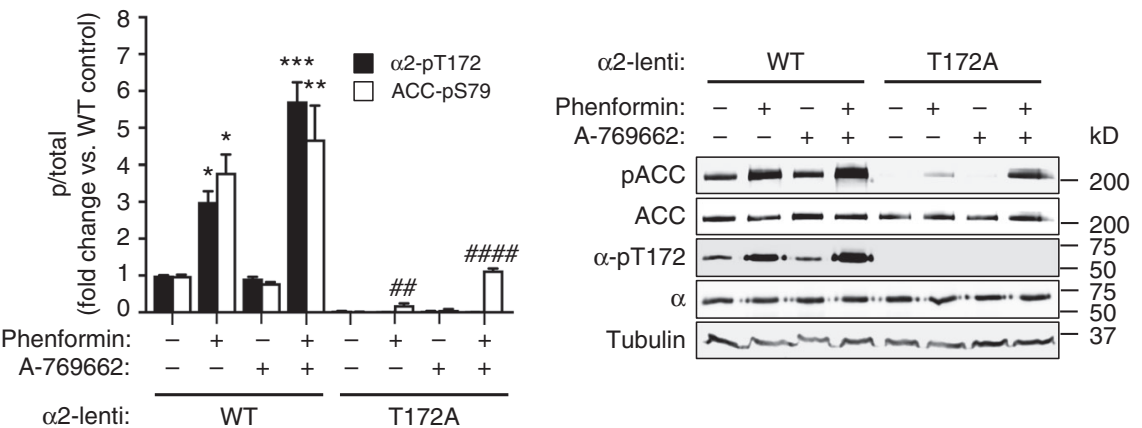

b
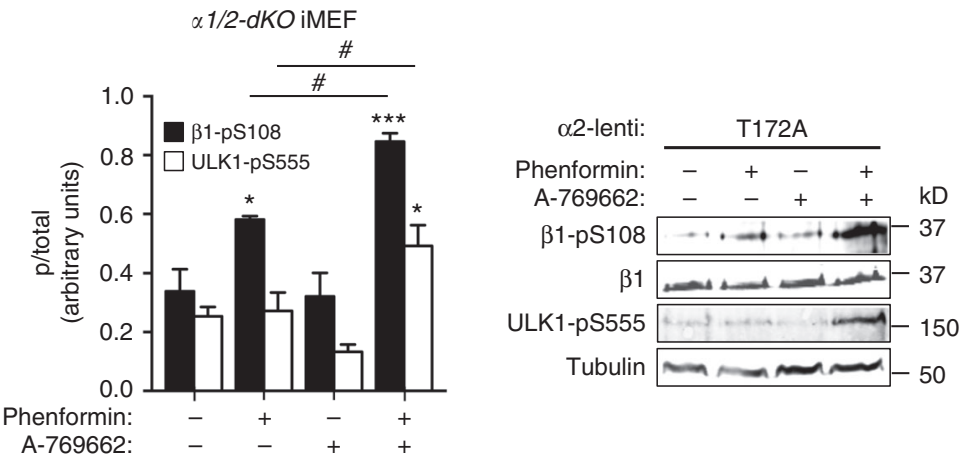

Fig. 8 Cellular AMPK signaling occurs independently of $\alpha 1-p$ Thr172. Statistical analyses were performed using one-way ANOVA with post hoc Dunnett's multiple comparison test. a Immunoblots for $\alpha 2$-pThr172 and pACC from $\alpha 1 / 2-d K O$ iMEFs-expressing $\alpha 2$ WT or T172A mutant, stimulated with 2 mM

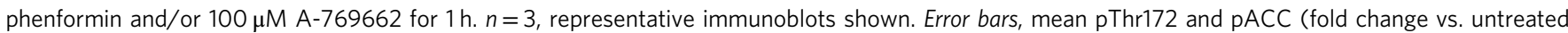

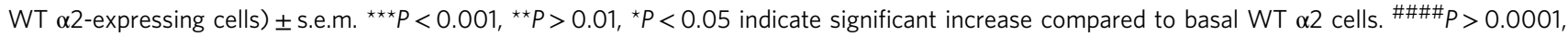
$\# \#<0.01$ indicate significant increase compared to basal $\alpha 2(T 172 A)$ cells. b Immunoblots for $\beta 1$-pSer108 and ULK1-pSer555 from $\alpha 1 / 2-d K O$ iMEFs-

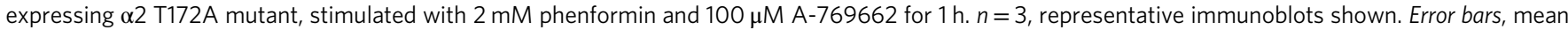
Ser108 and Ser555 phosphorylation (arbitrary units) \pm s.e.m. ${ }^{\star \star \star} P<0.001,{ }^{\star} P<0.05$ indicate significant increase compared to basal. ${ }^{\#} P<0.05$ indicates significant increase compared to phenformin treated

upstream kinases and protein targets located at intracellular membranes $^{3}, 30,31$. We examined the requirement for $\beta$ myristoylation in directing Ser108 phosphorylation during cellular metabolic stress. We found that KI- $\alpha 1 \beta 1 \gamma 1$ containing a myristoylation-deficient $\beta 1-\mathrm{G} 2 \mathrm{~A}$ mutant (non-myr) was insensitive to phenformin- or glucose starvation-induced phosphorylation of Ser108 in HEK293T cells (Fig. 7d). We examined the effect of $\beta$-myristoylation on cell-free ULK1-phosphorylation of Ser108 using myristoylated and non-myristoylated forms of KI- $\alpha 1 \beta 1 \gamma 1$. In contrast to our observation in cells, $\beta$-myristoylation resulted in significant suppression of ULK1-mediated Ser108 phosphorylation, which was not relieved by addition of AMP (Fig. 7e).

pThr172 is not absolutely required for AMPK signaling. To assess whether AMPK cellular signaling can be triggered independently of Thr172 phosphorylation, we expressed FLAGtagged $\alpha 2 \mathrm{WT}$ or T172A mutant in $\alpha 1 / 2-d K O$ iMEFs at similar levels (Fig. 8a). The T172A mutant possesses negligible basal and AMP-stimulated activities, but importantly can be sensitized to $\mathrm{ADaM}$ site metabolites/drugs through Ser108 phosphorylation ${ }^{13}$. In $\alpha 1 / 2-d K O$ iMEFs-expressing WT $\alpha 2$, phenformin, but not A-769662, induced robust increases in pThr172 and pACC from a high basal level. In cells expressing $\alpha 2(\mathrm{~T} 172 \mathrm{~A})$, phenformin and A-769662, either alone or in combination, failed to induce Thr172 phosphorylation as expected. Phenformin, but not A-769662, induced a small increase in pACC from an undetectable basal level. Phenformin/A-769662 co-incubation resulted in a further 5.8 -fold increase in pACC compared to phenformin alone, producing $>30 \%$ the pACC signal in WT $\alpha 2$-expressing cells treated with phenformin (Fig. 8a). Neither of these effects on pACC were detected in $\alpha 1 / 2-d K O$ iMEFs transduced with empty lentivirus (Supplementary Fig. 6), confirming that AMPK was the only kinase phosphorylating ACC-Ser79 under these conditions. A similar phosphorylation profile was seen for Ser108 in $\alpha 2$ (T172A) cells, with phenformin inducing a small increase that was amplified in the additional presence of A-769662 (Fig. 8b). Phenformin/A-769662 co-incubation was the only condition that induced a detectable increase in ULK1-Ser555 phosphorylation, an AMPK substrate that is important for ULK1 activation ${ }^{32}$ (Fig. 8b).

\section{Discussion}

In this study, we demonstrate that $\beta 1$-Ser108 in AMPK, a central co-ordinator of energy homeostasis, is a phosphorylation target for ULK1, a major regulator of autophagy initiation. Consequently, ULK1 sensitizes AMPK to A-769662 and salicylate, the active metabolic break-down product of acetylsalycilic acid (aspirin), independently of Thr172 phosphorylation (Fig. 4d). Salicylate stimulates fat utilization and reduces plasma fatty acids in vivo ${ }^{15}$, reduces de novo lipogenesis in human hepatocytes and $\mathrm{MEFs}^{33}, 34$, and reduces fatty acid and sterol synthesis in macrophages ${ }^{35}$. Daily aspirin prophylaxis is also associated with oncosuppression, an effect mirrored by the indirect AMPK activator metformin. Other $\beta 1$-AMPK-specific direct activators MT63-78 36 and the indole acid derivative PF-06409577 37 have shown promise as treatments for either prostate cancer or diabetic neuropathy, respectively. Although not investigated, Ser108 phosphorylation is likely a requirement for 
AMPK activation by these compounds; MT63-78 shares common structural features with A-769662, whereas the crystal structure of PF-06409577 bound to the ADaM site contains the phosphomimetic residue Asp instead of Ser108. Our finding that ULK1 phosphorylates Ser108 is significant given the emergence of this modification as a vital mechanistic constant for AMPK drugs. Discovery of a mechanism that induces AMPK drug sensitization independently of autophosphorylation also provides a potential strategy to treat non-small-cell lung and cervical carcinomas, associated with genetic loss of $\mathrm{LKB}^{38}$.

Accumulated evidence now depicts the $\mathrm{ADaM}$ site as an "orphan" allosteric site for an unidentified endogenous AMPK ligand: (i) $\beta 1$-Ser108 and contacting $\alpha$-residues are highly conserved among eukaryotes; (ii) the $\beta 1-\mathrm{ADaM}$ site is transient and regulated; (iii) both ULK1 phosphorylation of Ser108, and activation of AMPK by small molecule drugs, share a common specificity for the $\beta 1$-isoform; (iv) synergistic activation of unphosphorylated AMPK, orchestrated across all three AMPK subunits and topographically distant $\beta 1-\mathrm{ADaM}$ and $\gamma$-sites ${ }^{13}$, appears a highly intrinsic activation mechanism. Additionally, independent studies have described a disconnect between AMPK signaling (using elevated pACC as an index) and apparent AMPK activation (no increase in pThr172), most commonly in examinations of the role of skeletal muscle AMPK during exercise ${ }^{39-42}$, but also in response to reactive oxygen species (HeLa cells) ${ }^{43}$ and berberine (LAMTOR1-KO MEFs) ${ }^{44}$. We now demonstrate that significant cellular AMPK signaling can be triggered independently of pThr172 (Fig. 8a), although we are unable to determine whether this arises exclusively by Ser108 phosphorylation, $\mathrm{AMP} /$ drug synergistic activation of unphosphorylated enzyme, or a combination of both.

Applying quantitative phosphoproteomics to investigate a physiological role for Ser 108 phosphorylation, we identified significant differences in the phosphorylation profiles of several cell cycle-associated proteins in response to the phosphomimetic mutant $\beta 1$-S108E (Fig. 2). Our approach was limited in that we do not know whether potential metabolite(s) acting at the S108Estabilized $\mathrm{ADaM}$ site were elevated in response to phenformin, thus our findings are likely an underestimation of the global effects of Ser108 phosphorylation. It should also be emphasized that the differences reflect both primary and downstream phosphorylation events, so that the phosphosites detected encompass more than direct AMPK sites. None of these phosphorylated residues have been identified as direct AMPK substrates, however, both DOCK7_Ser452 and NHRF1_Ser285 are potential candidates based on consensus with the AMPK substrate recognition motif (Supplementary Table 1). $\beta 1-S 108 \mathrm{E}$ expression induced a significant increase in downstream phosphorylation of the PAK2 regulatory domain residue Ser141, an event required for full kinase activity and subsequent induction of the cytostatic and anti-apoptotic functions of full-length PAK2 ${ }^{45}, 46$. Other downstream targets are associated with regulation of cell cycle arrest (Bin1; GO:0071156), p53-mediated cell cycle arrest (TB182; GO:0006977) and cell division (CND1; GO:0051301). Although functional roles for the majority of sites detected in our analysis are unknown, our findings with PAK2 in particular point to a role for $\beta 1$-Ser108 phosphorylation in promoting energy stressinduced pro-survival pathways over cell death pathways. This model is consistent with other autophagy-inducing roles for AMPK, and Ser108-phosphorylation as a direct mechanism to activate AMPK by the autophagy initiator ULK1. Future studies will delineate the cellular signaling mechanisms specific to $\beta 1$-Ser108 phosphorylation, and bridge the gap between AMPK and the other identified downstream substrates.

Closer examination of our results from MEFs expressing only $\alpha 2(\mathrm{~T} 172 \mathrm{~A}) \mathrm{AMPK}$ provides further insight into ULK regulation

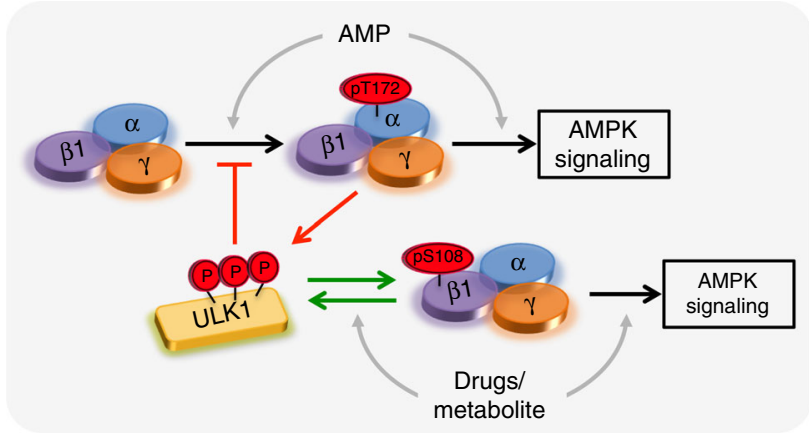

Fig. 9 An integrated model for ULK1 regulation of $\beta 1-A M P K$ signaling. The initial ULK stimulus (e.g., Ser555 phosphorylation) is provided by AMPK, activated itself in response to energy stress and elevated AMP:ATP. Once activated, ULK1 suppresses Thr172 phosphorylation and AMPK sensitivity to AMP via negative feedback (red arrows) ${ }^{20}$. ULK1 simultaneously phosphorylates Ser108, sensitizing AMPK to drugs/metabolites acting at the ADaM site. This would promote AMP-independent AMPK signaling and maintain ULK1 activity via positive feedback (green arrows). In our cell model, activity of $\alpha 2$ (T172A) AMPK is not elevated in response to AMP and requires additional stimulation with $A-769662$ to achieve ULK1 phosphorylation

of $\beta 1$-AMPK signaling (Fig. 8). An integrated model, involving positive and negative feedback loops, is shown in Fig. 9. AMPK signaling in these cells is hampered by insensitivity to AMP; consequently phenformin is unable to stimulate ULK1 activity, contributing to the weak pSer108 response (Fig. 8b). Further addition of A-769662 was sufficient to rescue AMPK activity and stimulate phosphorylation of ULK1-Ser555 and Ser108, potentially via positive feedback. cis-autophosphorylation of Ser108 in the $\alpha 2(\mathrm{~T} 172 \mathrm{~A})$ mutant is unlikely since activity of the unphosphorylated complex can only be achieved synergistically; in the AMP/drug-bound state Ser108 is presumably sequestered to the $\mathrm{ADaM}$ site and away from the AMPK active site. The close correlation between pACC, pSer108 and ULK-pSer555 profiles across all conditions provides support for a positive feedback mechanism between ULK and AMPK (Fig. 8a, b). Consistent with this, co-incubation of cells expressing only KI-AMPK with phenformin/A-769662 did not induce a further increase in pSer108, compared to phenformin treatment alone (Fig. 3d). Our model raises the intriguing prospect that, on ULK1-populated membranes (autophagosome, mitochondria), AMPK may be desensitized to AMP through suppression of $\alpha 1$-Thr172 phosphorylation, yet simultaneously sensitized to an alternate regulatory ligand through phosphorylation of $\beta 1$-Ser 108 .

Initial attempts to examine trans-phosphorylation of Ser108 in cells were confounded by strong background autophosphorylation signals in HEK293T endogenous AMPK, and high basal levels of pSer108 in immortalized MEFs (Fig. 6c). The cause of elevated pSer108 in iMEFs is unknown. Since KI-AMPK was not phosphorylated on Ser108 under basal conditions (Fig. 3d) we expect loss of a pSer108 phosphatase activity, leading to accumulation of Ser108 autophosphorylation in endogenous AMPK, may be a contributing factor. We were able to exploit the intramolecular "limitation" of Ser108 cis-autophosphorylation by using KI-AMPK in cells to uncouple background autophosphorylation from input by alternate signaling pathways. We identified conditions (glucose starvation, $\mathrm{H}_{2} \mathrm{O}_{2}$ and phenformin) under which Ser108 is phosphorylated independently of AMPK activity (Fig. 3b). These conditions also increase cellular LC3-II or ULK1-pSer555, both standard markers for autophagic activity ${ }^{47-49}$. Confirmation of ULK1-mediated phosphorylation of Ser108 in cells was provided by small molecule (Fig. 6a, b) or 
genetic (Fig. 6d) inhibition of ULK activity, each of which resulted in almost complete loss of stress-induced Ser108 trans-phosphorylation.

ULK1 phosphorylation of Ser108 is specific to the AMPK $\beta 1$-isoform (Fig. 5); this is likely due to sequence heterogeneity proximal to Ser108 in the $\beta 2$-isoform that diverges from the ULK consensus found in the corresponding $\beta 1$ residues $^{24}$. Interestingly, $\beta 1$ residues C-terminal to Ser108 (His109-Phe112) are considered unfavorable for ULK substrates. However, Ser108 is located at the end of a $\beta$-hairpin loop structure ${ }^{12}$ and $\mathrm{C}$-terminal residues may not participate in substrate recognition by ULK1. $\beta 1$-Ser108 does not appear to be a favorable substrate for ULK2 (Fig. 4b, c), although we cannot rule out the possibility that the two ULK2 preparations used in this study may have possessed inherently low activity, given the current lack of knowledge regarding cellular regulation and bona fide substrates for this kinase. Overall, our findings are in agreement with autophagy network mapping showing interaction with AMPK $\beta 1$ is restricted to ULK1, whereas ULK2 association is limited to AMPK $\beta 2^{41}$. Further investigation is warranted to determine subsets of ULK1 and ULK2 downstream targets in vivo.

Our results here, and those of others, provide strong evidence for potentiation of Ser108 phosphorylation by an AMP-myristoyl switch mechanism, in which AMP-induced conformational changes lead to ejection of the $\beta$-subunit myristoyl group from an intramolecular-binding site ${ }^{3}$. The exposed myristoyl group promotes AMPK targeting to intracellular membranes and, in this case, ULK1 co-localization. Cell treatments that promote pSer108 independently of autophosphorylation (glucose starvation, $\mathrm{H}_{2} \mathrm{O}_{2}$ and phenformin) (Fig. 3b) are associated with increased AMP/ ATP ratio, either through mitochondrial toxicity or disruption of ATP production ${ }^{50}$. Treatments that did not induce Ser108 phosphorylation activate AMPK independently of AMP (A769662, AICAR, ionomycin, amino-acid deprivation), or activate ULK independently of changes in adenylate charge (mTOR inhibitors) (Fig. 7a, c). The AMPK myristoyl-switching effect of ZMP (the metabolized product of AICAR and an AMP mimetic) has not yet been examined. Additionally, removal of either the AMP-sensing ability of AMPK with $\gamma 1(\mathrm{D} 245 \mathrm{~A})$ mutation (Fig. 7b), or $\beta$-subunit myristoylation with $\beta 1(\mathrm{G} 2 \mathrm{~A})$ mutation (Fig. 7d), abrogated glucose starvation- and/or phenformininduced Ser108 phosphorylation. $\beta 1(\mathrm{G} 2 \mathrm{~A})$ mutation was previously shown to suppress AMPK partitioning to intracellular membranes following glucose starvation ${ }^{3}$. ULK1 is also recruited to membrane structures during autophagy initiation, notably the autophagosome formation sites located near the $\mathrm{ER}^{51}$, and mitochondria ${ }^{52}$. ULK activity is associated with recruitment of other autophagy-associated proteins to the developing phagophore, including VPS34 and Beclin-1, both of which are phosphorylated by AMPK to achieve differential regulation of proand nonautophagy pathways ${ }^{53}$. Finally, $\beta$-myristoylation is strongly implicated in AMPK recruitment to LC3-containing puncta in the LKB1-deficient $\mathrm{H} 23$ human cancer cell line ${ }^{3 P}$. Collectively, these studies point to a close association of AMPK with ULK1 and the developing phagophore, and an important role for the exposed AMPK myristoyl group (myr switch ON) in targeting AMPK to phagophore membranes. A similar mechanism has been proposed to control AXIN-mediated AMPK recruitment to the late endosome/lysosome membrane for Thr172 phosphorylation and AMPK activation by LKB1 $1^{30}$. An unexpected finding was myristoyl-dependent suppression of Ser108 phosphorylation by ULK1 in cell-free assays (Fig. 7e), indicating that AMPK adopts a conformation in the myristoylburied state (myr switch OFF) that is unfavorable for ULK1 phosphorylation of Ser108. Repression of Ser108 phosphorylation was not alleviated by AMP; either this demonstrates a requirement for myristoyl-group membrane embedment, or an additional cellular component is required to derepress ULK phosphorylation through myristoyl group sequestration. These results may represent a protective mechanism to ensure Ser108 phosphorylation by ULK1 occurs exclusively at membrane surfaces.

In summary, we have identified an additional layer of communication between AMPK and ULK1, two major regulators of cellular energy homeostasis. ULK1 phosphorylation of Ser108, a post-translational modification important for AMPK drug action, may yield strategic opportunity to increase potency of AMPKtargeting therapeutics. Differential phosphorylation of AMPK at distinct cellular organelles/membranes raises the intriguing possibility of localized AMPK ligand sensitization. Myristoylswitching is a consistent driving force for both mechanisms; whether secondary targeting signals are required to partition distinct AMPK complexes between organelles represents an area of great interest for the future.

\section{Methods}

Reagents. DNA oligos were from Sigma (Supplementary Table 3). Antibodies for pan AMPK $\alpha$ (\#2793, clone F6, 1:1000 dilution), FLAG (\#2368, 1:1000 dilution), myc (\#2276, clone 9B11, 1:1000 dilution), HA (\#2367, clone 6E2, 1:1000 dilution), PAK1/2/3 (\#2604, 1:1000 dilution), ULK1 (\#4773, clone R600, 1:1000 dilution) and tubulin (\#3873, clone DM1A, 1:1000 dilution), and phosphospecific antibodies for AMPK $\alpha$-pThr172 (\#2535, clone 40H9, 1:1000 dilution), AMPK $\beta 1$-pSer108 (\#4181, 1:1000 dilution), ACC-pSer79 (\#3661, 1:1000 dilution), PAK1/2-pSer144/ Ser141 (\#2606, 1:1000 dilution), ULK1-pSer555 (\#5869, clone D1H4, 1:1000 dilution), and ULK1-pSer757 (\#6888, 1:1000 dilution) were from Cell Signaling Technology. AMPK- $\beta 1$ antibody (\#ab58175, 1:1000 dilution), A-769662 (\#ab120335) and AICAR were from Abcam. IRDye 680RD- or 800CW-labeled anti-immunoglobulin $\mathrm{G}$ antibodies (1:10,000 dilution) and IRDye 680RD-labeled streptavidin (1:20,000 dilution) were from LI-COR Biosciences. Glutathione Sepharose 4B and Streptavidin Sepharose high performance were from GE Life Sciences. FLAG synthetic peptide (DYKDDDK) was provided by GL Biochem (Shanghai). Other synthetic peptides were from Purar Chemicals. All peptides were purified by reversed-phase chromatography and stored as lyophilized powder. ULK2 recombinant protein was from Abcam. SBI-0206965, AZD8055 and INK128 were from ApexBio. FuGENE HD transfection reagent was from Promega Corporation. All other reagents were from Sigma.

Cell culture. COS7 and HEK293T cell lines were purchased from American Type Culture Collection. All cell lines were maintained in Dulbecco's modified Essential medium (DMEM) containing 10\% fetal bovine serum and antibiotics (penicillin, streptomycin) at $37^{\circ} \mathrm{C}$ with $5 \% \mathrm{CO}_{2}$. To generate iMEF cell lines, MEFs were extracted from WT or homozygous AMPK $\beta 1 \beta 2$ null embryos (days 12-14 post-coitum), generated by crossing homozygous $\beta 1$ and $\beta 2$ null mice ${ }^{54}$. WT and AMPK $\beta 1 / 2$ double knockout $(\beta 1 / 2-d K O)$ MEFs were immortalized by Fugene HD-mediated transfection with an SV40 large-T antigen expression construct. AMPK $\alpha 1 / 2$ double knockout $(\alpha 1 / 2-d K O)$ and ULK1/2 double knockout $(u l k 1 / 2-d K O)$ iMEFs were described previously ${ }^{23,} 29$.

Protein expression constructs. All mutants were generated using QuikChange site-directed mutagenesis kits (Stratagene). All constructs were sequence verified. Mammalian cell expression constructs were gifts from Reuben Shaw (pcDNA3 mouse FLAG-ULK1 (Addgene \#27636) and pcDNA3 mouse FLAG-ULK2 (Addgene \#27637)), Noboru Mizushima (pME18s-3xHA-human FIP200 (Addgene \#24303)) and Do-Hyung Kim (pRK5 human myc-Atg13 (Addgene \#31965)). SV40 large-T antigen expression construct pBSSVD2005 was a gift from David Ron (Addgene plasmid \#21826). Complementary DNA (cDNAs) for human AMPK $\alpha 1$ or $\alpha 2$ were generated with N-terminal FLAG-tag and cloned into pcDNA3 using XhoI/EcoRI (AMPK FLAG- $\alpha 1$ ) or XhoI/HindIII (AMPK FLAG- $\alpha 2$ ) restriction sites. Lentivirus expression constructs LeGO-iG2, second generation viral packaging vector $\mathrm{psPax} 2$ and ecotropic envelope vector $\mathrm{pHCMV}$-EcoEnv were gifts from Carl Walkley (St Vincent's Institute of Medical Research). cDNAs for human AMPK $\beta 1$ (WT and mutants), $\alpha 1$ (WT and kinase inactive D141A mutant), and $\alpha 2$ (WT and T172A mutant) were generated with C-terminal FLAG-tag and cloned into LeGO-iG2 using BamHI/NotI ( $\beta 1)$ or EcoRI/NotI ( $\alpha 1$ and $\alpha 2)$ restriction sites.

Protein expression and purification. Heterotrimeric human AMPK $(\alpha 1 \beta 1 \gamma 1$ and $\alpha 1 \beta 2 \gamma 1$ expressed as $\mathrm{N}$-terminal $\mathrm{His}_{6}-\alpha$ fusions; WT or mutants as indicated) was expressed in E. coli strain Rosetta (DE3) and purified using nickel-Sepharose and size exclusion chromatography as described previously ${ }^{13}$. Heterotrimeric human AMPK $(\alpha 1 \beta 1 \gamma 1$ and $\alpha 1 \beta 2 \gamma 1$ expressed as an N-terminal GST- $\alpha$ fusion; WT or mutants as indicated) was expressed in HEK293T cells as described previously ${ }^{3}$. 
Briefly, HEK293T cells at $40 \%$ confluency were triply transfected with expression constructs for AMPK $\alpha$-, $\beta$-, and $\gamma$-subunits, using transfection reagent FuGENE $\mathrm{HD}$ according to the manufacturer's protocols. Cells were harvested $48 \mathrm{~h}$ posttransfection in ice cold lysis buffer $(50 \mathrm{mM}$ Tris- $\mathrm{HCl}(\mathrm{pH} 7.4), 150 \mathrm{mM} \mathrm{NaCl}$, $50 \mathrm{mM} \mathrm{NaF}, 1 \mathrm{mM}$ sodium pyrophosphate, $1 \mathrm{mM}$ EDTA, $1 \mathrm{mM} \mathrm{EGTA}, 1 \%(\mathrm{v} / \mathrm{v})$ Triton X-100, protease inhibitors). Lysates were clarified by centrifugation at $14,000 \mathrm{rpm}$ for $5 \mathrm{~min}$ and flash frozen in liquid $\mathrm{N}_{2}$ until processing. AMPK was purified on glutathione Sepharose 4B and eluted in $50 \mathrm{mM}$ Tris- $\mathrm{HCl}$, (pH 7.4), $100 \mathrm{mM} \mathrm{NaCl}, 10 \%$ glycerol supplemented with $20 \mathrm{mM}$ glutathione. ULK1/2 and ULK1/FIP200/Atg13 complex was expressed by FuGENE HD transient transfection of HEK293T cells. Lysates were harvested after $48 \mathrm{~h}$, clarified by centrifugation and ULK purified on anti-FLAG M2 affinity gel. ULK was eluted in $50 \mathrm{mM}$ Tris- $\mathrm{HCl}$ (pH 7.4), $100 \mathrm{mM} \mathrm{NaCl}, 10 \%$ glycerol, $0.01 \%$ Tween-20 supplemented with $1 \mathrm{mg} / \mathrm{ml}$ FLAG peptide. CaMKK2 isoform 1 was produced in Sf21 insect cells as a C-terminal FLAG fusion as described previously ${ }^{3}$. Briefly, Sf21 cells were infected at a multiplicity of infection of 10 and harvested $72 \mathrm{~h}$ post-infection. Cell lysates were prepared as for recombinant AMPK, and protein was purified on antiFLAG M2 affinity gel. CaMKK2 was eluted in $50 \mathrm{mM}$ Tris- $\mathrm{HCl}$ (pH 7.4), $100 \mathrm{mM}$ $\mathrm{NaCl}, 10 \%$ glycerol, $0.01 \%$ Tween-20 supplemented with $1 \mathrm{mg} / \mathrm{ml}$ FLAG peptide.

\section{Lentiviral-mediated AMPK expression and purification. Ecotropic lentivirus} was generated by transient transfection of HEK293T cells using calcium phosphate. Briefly, 1 day prior to transfection, $2-2.5 \times 10^{6}$ HEK293T cells were seeded per $10 \mathrm{~cm}$ culture dish. LeGO-iG2, psPax2 and pHCMV-EcoEnv plasmids $(10 \mu \mathrm{g}$, $6.3 \mu \mathrm{g}$ and $3.8 \mu \mathrm{g}$ per $10 \mathrm{~cm}$ culture dish, respectively) were mixed together with $2 \mathrm{M} \mathrm{CaCl}_{2}$ solution $(244 \mathrm{mM}$ final concentration in $500 \mu \mathrm{l})$. The DNA/CaCl solution was added drop-wise, with vortexing, into $500 \mu \mathrm{l}$ of 2 xHEPES-buffered saline, $\mathrm{pH}$ 7.06. After $20 \mathrm{~min}$ incubation at RT, the mixture was transferred dropwise onto HEK293T cell culture and incubated. Within $16 \mathrm{~h}$ of transfection, cell were washed with phosphate-buffered saline (PBS) and replaced with $6 \mathrm{ml}$ fresh media. The lentivirus-containing supernatant was harvested after 48 and $72 \mathrm{~h}$ posttransfection and stored at $-80^{\circ} \mathrm{C}$.

iMEFs were transduced in 6-well plates by spinoculation. Briefly, 1 day prior to transduction, $0.5 \times 10^{5}$ cells per well in a 6 -well plate were seeded in $2 \mathrm{ml}$ media. $200 \mu \mathrm{l}$ lentivirus supernatant in the presence of $8 \mu \mathrm{g} / \mathrm{ml}$ polybrene in $2 \mathrm{ml}$ media was spun for $98 \mathrm{~min}$ at $25^{\circ} \mathrm{C}, 1100 \times \mathrm{g}$ (Heraeus Megafuge $2.0 \mathrm{R}$ ). Lentiviruscontaining media was replaced with an equal volume of fresh media after $24 \mathrm{~h}$. Seventy-two hourspost-transduction, iMEFs were incubated with fresh media for 1 $\mathrm{h}$ and treated as indicated. Cells were harvested by washing with ice-cold PBS, followed by rapid lysis in situ using $100 \mu \mathrm{l}$ ice-cold lysis buffer $(50 \mathrm{mM}$ Tris. $\mathrm{HCl}$ (pH 7.4), $150 \mathrm{mM} \mathrm{NaCl}, 50 \mathrm{mM} \mathrm{NaF}, 1 \mathrm{mM}$ sodium pyrophosphate, $1 \mathrm{mM}$ EDTA, $1 \mathrm{mM}$ EGTA, $1 \mathrm{mM}$ dithiothreitol, $1 \%$ (v/v) Triton X-100 and protease inhibitors), and cellular debris was removed by centrifugation.

FLAG-tagged AMPK $\alpha 1$ or $\alpha 2$, and acetyl-CoA carboxylase (ACC), were isolated from iMEF cell lysates using anti-FLAG M2 affinity gel or Streptavidin Sepharose high performance, respectively. Immobilized ACC was washed extensively with buffer A and eluted in $2 \times$ sodium dodecyl sulfate polyacrylamide gel electrophoresis (SDS-PAGE) loading sample buffer for immunoblotting.

Kinase activity assays. ULK and AMPK activities were determined by phosphorylation of a synthetic peptide (S108tide: KLPLTRSHNNFVARRR, corresponding to AMPK $\beta 1(102-114)$ with three additional C-terminal Arg residues to promote binding to P81 phosphocellulose paper) using $200 \mu \mathrm{M}\left[\gamma^{3}{ }^{32} \mathrm{P}\right] \mathrm{ATP}$ and $5 \mathrm{mM} \mathrm{MgCl}_{2}$ in a $25 \mu \mathrm{l}$ reaction volume at $30^{\circ} \mathrm{C}$. Reactions were terminated after 10 min by spotting $15 \mu \mathrm{l}$ onto P81 phosphocellulose paper (Whatman) and washing in $1 \%$ phosphoric acid. Radioactivity was quantified by scintillation counting.

Kinase screen. Kinase screening (IKPT service) was performed by Kinexus, Canada. S108tide $(100 \mu \mathrm{M})$ was screened against a panel of 284 selected Ser/Thr kinases with single replicate. Most assays were performed for $15 \mathrm{~min}$ duration, $50 \mu \mathrm{M}\left[\gamma_{-}{ }^{33} \mathrm{P}\right] \mathrm{ATP}$, in a $25 \mu \mathrm{l}$ reaction volume at $30^{\circ} \mathrm{C}$.

Generation of AMPK $\beta 2$-pSer108 phosphospecific antibody. A phosphorylated synthetic peptide (CSTKIPLIKpSHNDFVAILD, corresponding to AMPK $\beta 2$ (100-117)) was coupled to keyhole limpet hemocyanin via the peptide N-terminal cysteine residue using the coupling reagent $N$-succinimidyl-3(-2-pyridyldithio) propionate. Rabbits were immunized with $2 \mathrm{mg}$ of peptide conjugate initially in $50 \%(\mathrm{v} / \mathrm{v})$ Freunds complete adjuvant and in 50\% (v/v) Freunds incomplete adjuvant for subsequent immunizations. Rabbits were boosted fortnightly with 2 $\mathrm{mg}$ of peptide conjugate and bled 7 days after booster injections. The pSer108 antibody was then purified from serum by peptide affinity chromatography. Specificity for $\beta 2$-pSer108 was evaluated by immunoblot against CaMKK2phosphorylated AMPK $\alpha 1 \beta 2 \gamma 1$, and $\alpha 1 \beta 1 \gamma 1$.

Immunoblotting. Samples were electrophoresed by $12 \%$ SDS-PAGE and transferred to Immobilon FL polyvinylidine-flouride membrane (Millipore). The membrane was blocked with $2 \%$ nonfat dry milk in PBS $+0.1 \%$ Tween 20 (PBS-T) for $1 \mathrm{~h}$ at room temperature. Membranes were incubated either overnight or for $1 \mathrm{~h}$ with $1^{\circ}$ antibodies (diluted 1:500-1:2000 in PBS-T), prior to 30 min incubation with anti-rabbit or anti-mouse IgG $2^{\circ}$ antibody fluorescently labeled with IR680 or IR800. Immunoreactive bands were visualized on an Odyssey membrane imaging system (LI-COR Biosciences) and densitometry analyses performed using integrated software. Uncropped western blot images associated with this study are presented in Supplementary Fig. 7.

Quantitative global and phosphoproteomic analysis. $\beta 1 / 2-d K O$ iMEFs, transduced with AMPK $\beta 1-S 108 \mathrm{~A}$ or $\beta 1-\mathrm{S} 108 \mathrm{E}$, as described above, were incubated with $2 \mathrm{mM}$ phenformin for $1 \mathrm{~h}$. Treated lysates were acetone $\left(-20^{\circ} \mathrm{C}\right)$ precipitated and resuspended in $8 \mathrm{M}$ urea/50 $\mathrm{mM}$ triethyl ammonium bicarbonate (TEAB) solution. Protein lysates were reduced with $10 \mathrm{mM}$ tris(2-carboxyethyl)phosphine (TCEP) for $45 \mathrm{~min}$ at $37^{\circ} \mathrm{C}$, and alkylated with $55 \mathrm{mM}$ iodoacetamide for $30 \mathrm{~min}$ at room temperature in the dark. Samples were diluted to $1 \mathrm{M}$ urea in $25 \mathrm{mM}$ TEAB and trypsin $(1: 60, \mathrm{w} / \mathrm{w})$ digested overnight at $37^{\circ} \mathrm{C}$. Digested tryptic peptides were cleaned up using Oasis HLB (hydrophilic lipophilic balance) solid phase extraction (SPE) cartridges (Waters) and freeze-dried overnight. Resuspended tryptic digests were used for stable-isotope dimethyl labeling as described previously ${ }^{5}$. $\beta 1$-S108A and $\beta 1$-S108E derived tryptic peptides were used for light and medium labeling, respectively. Labeled peptides were mixed (1:1) and an aliquot (15 $\mu \mathrm{l})$ was taken out and run on liquid chromatography-mass spectrometry (LC-MS)/MS for total proteome changes. Remaining mixed labeled peptides were SPE cleaned up and freeze-dried before used for $\mathrm{TiO}_{2}$ phosphopeptides enrichment. Phosphopeptide enrichment by $\mathrm{TiO}_{2}$ microcolumns was carried out as described previously ${ }^{56}$ Mixed labeled peptides and $\mathrm{TiO}_{2}$ enriched phosphopeptides were analyzed by LCMS/MS using a Q-Exactive plus mass spectrometer (Thermo Scientific) fitted with nanoflow reversed-phase-high-performance liquid chromatography (HPLC) (Ultimate 3000 RSLC, Dionex). The nano-LC system was equipped with an Acclaim Pepmap nano-trap column (Dionex-C18, $100 \AA 7,75 \mu \mathrm{m} \times 2 \mathrm{~cm}$ ) and an Acclaim Pepmap RSLC analytical column (Dionex-C18, $100 \AA$, $75 \mu \mathrm{m} \times 50 \mathrm{~cm}$ ). Typically for each LC-MS/MS experiment, $5 \mu$ of the peptide mix was loaded onto the enrichment (trap) column at an isocratic flow of $5 \mu \mathrm{l} / \mathrm{min}$ of $3 \% \mathrm{CH}_{3} \mathrm{CN}$ containing $0.1 \%$ formic acid for $6 \mathrm{~min}$ before the enrichment column is switched in-line with the analytical column. The eluents used for the LC were $0.1 \% \mathrm{v} / \mathrm{v}$ formic acid (solvent $\mathrm{A}$ ) and $100 \% \mathrm{CH}_{3} \mathrm{CN} / 0.1 \%$ formic acid $\mathrm{v} / \mathrm{v}$ (solvent $\mathrm{B}$ ). The gradient used was $3 \%$ B to $25 \%$ B for $23 \mathrm{~min}, 25 \%$ B to $40 \%$ B in 2 min, $40 \%$ B to $80 \% \mathrm{~B}$ in $2 \mathrm{~min}$ and maintained at $85 \% \mathrm{~B}$ for the final $2 \mathrm{~min}$ before equilibration for $9 \mathrm{~min}$ at $3 \% \mathrm{~B}$ prior to the next analysis. All spectra were acquired in positive mode with full scan MS spectra scanning from $\mathrm{m} / \mathrm{z} 375-1400$ at 70,000 resolution with AGC target of $3 \mathrm{e} 6$ with maximum accumulation time of $50 \mathrm{~ms}$. Lockmass of 445.120024 was used. The 15 most intense peptide ions with charge states $\geq 2-5$ were isolated with isolation window of $1.2 \mathrm{~m} / \mathrm{z}$ and fragmented with normalized collision energy of 30 at 35,000 resolution with AGC target of $1 \mathrm{e} 5$ with maximum accumulation time of $120 \mathrm{~ms}$. Underfill threshold was set to $2 \%$ for triggering of precursor for MS2. Dynamic exclusion was activated for $30 \mathrm{~s}$. Mass spectrometric raw data were processed and analyzed using Proteome Discoverer 2.1 (Thermo Scientific) with Mascot search algorithm against mouse SwissProt database. Perseus 1.5.6.0 was used for further data analysis ${ }^{57}$. IPA software (QIAGEN Redwood City) was used for network and pathway analysis.

In-gel digestion of protein bands and mass spectrometry. SDS-PAGE protein bands were excised and simultaneously reduced and alkylated with $10 \mathrm{mM}$ TCEP and $40 \mathrm{mM} 2$-choloracetamide for $1 \mathrm{~h}$ at room temperature. Proteins were digested with $13 \mathrm{ng} / \mu \mathrm{l}$ of sequencing grade trypsin (Promega) overnight at $37^{\circ} \mathrm{C}$ and peptides desalted using in-house made microC18 columns ( $3 \mathrm{M}$ empore). Peptides were resuspended in $0.1 \%$ formic acid, $5 \%$ acetonitrile and analyzed on a Dionex 3500RS nanoUHPLC coupled to an Orbitrap Fusion mass spectrometer with Tune v2.0.1258 in positive mode. Peptides were separated using an in-house packed $75 \mu \mathrm{m} \times 40 \mathrm{~cm}$ pulled column (1.9 $\mu \mathrm{m}$ particle size, C18AQ; Dr Maisch, Germany) with a gradient of $2-30 \%$ acetonitrile containing $0.1 \% \mathrm{FA}$ over $60 \mathrm{~min}$ at $250 \mathrm{nl} /$ min at $55^{\circ} \mathrm{C}$. An MS1 scan was acquired from $350-1550$ (120,000 resolution, $5 \mathrm{e}^{5}$ AGC, $100 \mathrm{~ms}$ injection time) followed by MS/MS data-dependent acquisition with HCD and detection in the Orbitrap (60,000 resolution, $2 \mathrm{e}^{5}$ AGC, 120 ms injection time, $40 \mathrm{NCE}, 2.0 \mathrm{~m} / \mathrm{z}$ quadrupole isolation width) and, EThcD and detection in the orbitrap (60,000 resolution, $2 \mathrm{e}^{5}$ AGC, $120 \mathrm{~ms}$ injection time, calibrated chargedependent ETD reaction times $[2+121 ; 3+54 ; 4+30 ; 5+20 ; 6+13 ; 7+; 10 \mathrm{~ms}]$, $25 \mathrm{NCE}$ for HCD supplemental activation, $2.0 \mathrm{~m} / z$ quadrupole isolation width). All raw data were analyzed with MaxQuant v1.5.3.25 $5^{58}$ and searched against the human UniProt database with default settings including phosphorylation of S, T, and $\mathrm{Y}$ as a variable modification and match between runs enabled.

Nucleotide measurements. Adenine nucleotides from HEK293T perchlorate extracts were measured by LC-MS on an ABISCIEX 5500 QTRAP mass spectrometer ${ }^{16}$. AEC was calculated from ratios of [AMP], [ADP], and [ATP] (Equation 1): 
Statistical analysis. The data are presented as mean values \pm s.e.m. of at least three independent experiments. The unpaired two-tailed Student's $t$-test was used for all comparisons unless stated.

Data availability. The mass spectrometry proteomics data have been deposited to the ProteomeXchange Consortium via the PRIDE ${ }^{59}$ partner repository with the data set identifier PXD006365. The authors declare that all other data supporting the findings of this study are available within the paper and its Supplementary Information files, or are available from the authors upon reasonable request.

Received: 27 September 2016 Accepted: 14 July 2017

Published online: 18 September 2017

\section{References}

1. Hardie, D. G. AMPK - sensing energy while talking to other signaling pathways. Cell Metab. 20, 939-952 (2014).

2. Steinberg, G. R. \& Kemp, B. E. AMPK in health and disease. Physiol. Rev. 89, 1025-1078 (2009)

3. Oakhill, J. S. et al. $\beta$-Subunit myristoylation is the gatekeeper for initiating metabolic stress sensing by AMP-activated protein kinase (AMPK). Proc. Natl Acad. Sci. USA 107, 19237-19241 (2010).

4. Houde, V. P. et al. Investigation of LKB1 Ser431 phosphorylation and Cys433 farnesylation using mouse knockin analysis reveals an unexpected role of prenylation in regulating AMPK activity. Biochem. J. 15, 41-56 (2014).

5. Xiao, B. et al. Structure of mammalian AMPK and its regulation by ADP. Nature 472, 230-233 (2011).

6. Oakhill, J. S. et al. AMPK is a direct adenylate charge-regulated protein kinase. Science 332, 1433-1435 (2011)

7. Chen, L. et al. AMP-activated protein kinase undergoes nucleotide-dependent conformational changes. Nat. Struct. Mol. Biol. 19, 716-718 (2012).

8. Hawley, S. A. et al. Characterization of the AMP-activated protein kinase kinase from rat liver and identification of threonine 172 as the major site at which it phosphorylates AMP-activated protein kinase. J. Biol. Chem. 271, 27879-27887 (1996).

9. Scott, J. W. et al. CBS domains form energy-sensing modules whose binding of adenosine ligands is disrupted by disease mutations. J. Clin. Invest. 113, 274-284 (2004).

10. Langendorf, C. G. et al. Structural basis of allosteric and synergistic activation of AMPK by furan-2-phosphonic derivative C2 binding. Nat. Commun. 7, 10912 (2016).

11. Sanders, M. J. et al. Defining the mechanism of activation of AMP-activated protein kinase by the small molecule A-769662, a member of the thienopyridone family. J. Biol. Chem. 282, 32539-32548 (2007).

12. Xiao, B. et al. Structural basis of AMPK regulation by small molecule activators. Nat. Commun. 4, 3017 (2013)

13. Scott, J. W. et al. Small molecule drug A-769662 and AMP synergistically activate naïve AMPK independent of upstream kinase signaling. Chem. Biol. 21, 619-627 (2014)

14. Calabrese, M. F. et al. Structural basis for AMPK activation: natural and synthetic ligands regulate kinase activity from opposite poles by different molecular mechanisms. Structure 22, 1161-1172 (2014).

15. Hawley, S. A. et al. The ancient drug salicylate directly activates AMP-activated protein kinase. Science 336, 918-922 (2012).

16. Scott, J. W. et al. Inhibition of AMP-activated protein kinase at the allosteric drug-binding site promotes islet insulin release. Chem. Biol. 22, 705-711 (2015).

17. Scott, J. W. et al. Thienopyridone drugs are selective activators of AMP-activated protein kinase betal-containing complexes. Chem. Biol. 15, 1220-1230 (2008).

18. Oakhill, J. S., Scott, J. W. \& Kemp, B. E. AMPK functions as an adenylate charge-regulated protein kinase. Trends Endocrinol. Metab. 23, 125-132 (2012).

19. Suzuki, T. et al. Inhibition of AMPK catabolic action by GSK3. Mol. Cell 50, 407-419 (2013)

20. Löffler, A. S. et al. Ulk1-mediated phosphorylation of AMPK constitutes a negative regulatory feedback loop. Autophagy 7, 696-706 (2011).

21. Mitchelhill, K. I. et al. Posttranslational modifications of the 5'-AMP-activated protein kinase $\beta 1$ subunit. J. Biol. Chem. 272, 24475-24479 (1997).

22. O'Neill, H. M. et al. AMP-activated protein kinase (AMPK) betalbeta2 muscle null mice reveal an essential role for AMPK in maintaining mitochondrial content and glucose uptake during exercise. Proc. Natl Acad. Sci. USA 108, 16092-16097 (2011)

23. Laderoute, K. R. et al. 5-AMP-activated protein kinase (AMPK) is induced by low-oxygen and glucose deprivation conditions found in solid-tumor microenvironments. Mol. Cell. Biol. 26, 5336-5347 (2016).

24. Egan, D. F. et al. Small molecule inhibition of the autophagy kinase ULK1 and identification of ULK1 substrates. Mol. Cell 59, 285-297 (2015).

25. Truong, T. H. \& Carroll, K. S. Redox regulation of protein kinases. Crit. Rev. Biochem. Mol. Biol. 48, 332-356 (2013).
26. Erickson, J. R. et al. A dynamic pathway for calcium-independent activation of CaMKII by methionine oxidation. Cell 133, 462-474 (2008).

27. Humphries, K. M., Deal, M. S. \& Taylor, S. S. Enhanced dephosphorylation of cAMP-dependent protein kinase by oxidation and thiol modification. J. Biol. Chem. 280, 2750-2758 (2005).

28. Cosentino-Gomes, D., Rocco-Machado, N. \& Meyer-Fernandes, J. R. Cell signaling through protein kinase $\mathrm{C}$ oxidation and activation. Int. J. Mol. Sci. 13, 10697-10721 (2012).

29. Cheong, H., Lindsten, T., Wu, J., Lu, C. \& Thompson, C. B. Ammonia-induced autophagy is independent of ULK1/ULK2 kinases. Proc. Natl Acad. Sci. USA 108, 11121-11126 (2011).

30. Zhang, Y.-L. et al. AMP as a low-energy charge signal autonomously initiates assembly of AXIN-AMPK-LKB1 complex for AMPK activation. Cell Metab. 18, 546-555 (2013).

31. Liang, J. et al. Myristoylation confers noncanonical AMPK functions in autophagy selectivity and mitochondrial surveillance. Nat. Commun. 6, 7926 (2015).

32. Egan, D. F. et al. Phosphorylation of ULK1 (hATG1) by AMP-activated protein kinase connects energy sensing to mitophagy. Science 331, 456-461 (2011).

33. Ford, R. J. et al. Metformin and salicylate synergistically activate liver AMPK, inhibit lipogenesis and improve insulin sensitivity. Biochem. J. 468, 125-132 (2015).

34. O'Brien, A. J. et al. Salicylate activates AMPK and synergizes with metformin to reduce the survival of prostate and lung cancer cells ex vivo through inhibition of de novo lipogenesis. Biochem. J. 469, 177-187 (2015).

35. Fullerton, M. D. et al. Salicylate improves macrophage cholesterol homeostasis via activation of Ampk. J. Lipid Res. 56, 1025-1033 (2015).

36. Zadra, G. et al. A novel direct activator of AMPK inhibits prostate cancer growth by blocking lipogenesis. EMBO Mol. Med. 6, 519-538 (2014).

37. Cameron, K. O. et al. Discovery and preclinical characterization of 6-chloro-5[4-(1-hydroxycyclobutyl)phenyl]-1H-indole-3-carboxylic acid (PF-06409577), a direct activator of adenosine monophosphate-activated protein kinase (AMPK), for the potential treatment of diabetic nephropathy. J. Med. Chem. 59, 8068-8081 (2016).

38. Shackleford, D. B. \& Shaw, R. J. The LKB1-AMPK pathway: metabolism and growth control in tumour suppression. Nat. Rev. Cancer 9, 563-575 (2009).

39. Wojtaszewski, J. F. et al. Dissociation of AMPK activity and ACC $\beta$ phosphorylation in human muscle during prolonged exercise. Biochem. Biophys. Res. Commun. 298, 309-316 (2002).

40. Jørgensen, S. B. et al. Knockout of the alpha2 but not alpha1 5'-AMP-activated protein kinase isoform abolishes 5-aminoimidazole-4-carboxamide-1-beta-4ribofuranoside but not contraction-induced glucose uptake in skeletal muscle. $J$. Biol. Chem. 279, 1070-1079 (2004).

41. Dzamko, N. et al. AMPK-independent pathways regulate skeletal muscle fatty acid oxidation. J. Physiol. 586, 5819-5831 (2008).

42. Schwalm, C. et al. Activation of autophagy in human skeletal muscle is dependent on exercise intensity and AMPK activation. FASEB J. 29, 3515-3526 (2015).

43. Alexander, A. et al. ATM signals to TSC2 in the cytoplasm to regulate mTORC1 in response to ROS. Proc. Natl Acad. Sci. USA 107, 4153-4158 (2010).

44. Zhang, S.-C. et al. The lysosomal v-ATPase-ragulator complex is a common activator for AMPK and mTORC1, acting as a switch between catabolism and anabolism. Cell Metab. 20, 526-540 (2014).

45. Jung, J. H. \& Traugh, J. A. Regulation of the interaction of Pak2 with Cdc42 via autophosphorylation of serine 141. J. Biol. Chem. 280, 40025-40031 (2005).

46. Jakobi, R., Moertl, E. \& Koeppel, M. A. p21-activated protein kinase gammaPAK suppresses programmed cell death of BALB3T3 fibroblasts. J. Biol. Chem. 276, 16624-16634 (2001)

47. Kundu, M. et al. Ulk1 plays a critical role in the autophagic clearance of mitochondria and ribosomes during reticulocyte maturation. Blood 112, 1493-1502 (2008).

48. Mitter, S. K. et al. Dysregulated autophagy in the RPE is associated with increased susceptibility to oxidative stress and AMD. Autophagy 10, 1989-2005 (2014).

49. Behrends, C., Sowa, M. E., Gygi, S. P. \& Harper, J. W. Network organization of the human autophagy system. Nature 466, 68-76 (2010).

50. Hardie, D. G. AMPK: positive and negative regulation, and its role in wholebody energy homeostasis. Curr. Opin. Cell Biol. 33, 1-7 (2015).

51. Russell, R. C., Yuan, H. X. \& Guan, K. L. Autophagy regulation by nutrient signaling. Cell Res. 24, 42-57 (2014).

52. $\mathrm{Wu}, \mathrm{W}$. et al. ULK1 translocates to mitochondria and phosphorylates FUNDC1 to regulate mitophagy. EMBO Rep. 15, 566-575 (2014).

53. Kim, J. et al. Differential regulation of distinct Vps34 complexes by AMPK in nutrient stress and autophagy. Cell 152, 290-303 (2013).

54. Davies, M. et al. Novel mechanisms of $\mathrm{Na}^{+}$retention in obesity: phosphorylation of NKCC2 and regulation of SPAK/OSR1 by AMPK. Am. J. Physiol. Renal Physiol. 307, F96-F106 (2014). 
55. Boersema, P. J., Raijmakers, R., Lemeer, S., Mohammed, S. \& Heck, A. J. Multiplex peptide stable isotope dimethyl labeling for quantitative proteomics. Nat. Protoc. 4, 484-494 (2009).

56. Thingholm, T. E., Jorgensen, T. J., Jensen, O. N. \& Larsen, M. R. Highly selective enrichment of phosphorylated peptides using titanium dioxide. Nat. Protoc. 1, 1929-1935 (2006).

57. Tyanova, S. et al. The Perseus computational platform for comprehensive analysis of (prote)omics data. Nat. Methods 13, 731-740 (2016).

58. Cox, J. \& Mann, M. MaxQuant enables high peptide identification rates, individualized p.p.b.-range mass accuracies and proteome-wide protein quantification. Nat. Biotechnol. 26, 1367-1372 (2008).

59. Vizcaíno, J. A. et al. 2016 update of the PRIDE database and related tools. Nucleic Acids Res. 44, D447-D456 (2016).

\section{Acknowledgements}

This work was supported by grants from the Australian Research Council (ARC) and the National Health and Medical Research Council (NHMRC). G.R.S. is a Canada Research Chair in Metabolism and Obesity and the J. Bruce Duncan Chair in Metabolic Diseases. B.E.K. is an NHMRC Research Fellow. J.S.O. is an ARC Future Fellow. Supported in part by the Victorian Government's Operational Infrastructure Support Program.

\section{Author contributions}

T.A.D., N.X.Y.L., and J.S.O. designed and coordinated the study. T.A.D., N.X.Y.L., J.W.S., A.H., B.L.P., K.R.W.N., M.T.O., and C.G.L. performed the experiments. S.G., M.K., and B.V. provided immortalized mouse embryonic fibroblasts. J.W.S., G.R.S., K.S., and B.E.K. provided intellectual input. All authors contributed to writing the manuscript.

\section{Additional information}

Supplementary Information accompanies this paper at doi:10.1038/s41467-017-00628-y.

Competing interests: The authors declare no competing financial interests.

Reprints and permission information is available online at http://npg.nature.com/ reprintsandpermissions/

Publisher's note: Springer Nature remains neutral with regard to jurisdictional claims in published maps and institutional affiliations.

(c) (i) Open Access This article is licensed under a Creative Commons Attribution 4.0 International License, which permits use, sharing, adaptation, distribution and reproduction in any medium or format, as long as you give appropriate credit to the original author(s) and the source, provide a link to the Creative Commons license, and indicate if changes were made. The images or other third party material in this article are included in the article's Creative Commons license, unless indicated otherwise in a credit line to the material. If material is not included in the article's Creative Commons license and your intended use is not permitted by statutory regulation or exceeds the permitted use, you will need to obtain permission directly from the copyright holder. To view a copy of this license, visit http://creativecommons.org/ licenses/by/4.0/.

(C) The Author(s) 2017 